\title{
Existence of solutions and stability for impulsive neutral stochastic functional differential equations
}

\author{
Mimia Benhadria ${ }^{\mathrm{a}}$, Tomás Caraballo ${ }^{\mathrm{b}}$, Halim Zeghdoudic $^{\mathrm{c}}$ \\ a Frères Mentouri Constantine University, \\ Department of Mathematics, Algeria. \\ emails: mbenhadri@yahoo.com \\ ${ }^{\mathrm{b}}$ Departamento de Ecuaciones Difererenciales y Análisis Numérico, \\ Universidad de Sevilla c/ Tarfia s/n, 41012-Sevilla (Spain) \\ email: caraball@us.es \\ ${ }^{c}$ LaPS Laboratory, Badji-Mokhtar University, \\ Box 12, Annaba, 23000, Algeria. \\ email: halim.zeghdoudi@univ-annaba.dz
}

April 15, 2019

\begin{abstract}
In this paper we prove some results on the existence of solutions and the mean square asymptotic stability for a class of impulsive neutral stochastic differential systems with variable delays by using a contraction mapping principle. Namely, a sufficient condition ensuring the asymptotic stability is proved. The assumptions do not impose any restrictions neither on boundedness nor on the differentiability of the delay functions. In particular, the results improve some previous ones in the literature. Finally, an example is exhibited to illustrate the effectiveness of the results.
\end{abstract}

AMS Subject Classifications: 34K20, 34K13, 92B20

Keywords: Fixed points theory; Asymptotic stability in mean square; Neutral stochastic differential equations; Variable delays; Impulses. 


\section{Introduction}

Impulsive differential equations appear as a natural description of observed evolution phenomena in several real world problems. There are various good monographs on impulsive differential equations $[1,2,14,30]$. Many processes studied in applied sciences are represented by differential equations. However, the situation is quite different in many physical phenomena that have a sudden change in their states. For instance, mechanical systems with impact, population dynamics, mathematical economy, chemical technology, electric technology, chemistry, engineering, control theory, medicine etc.

The theory of impulsive differential systems is an important branch of the differential equations field. The first paper in this theory was published in the 60 's by A. D. Mishkis and V. D. Mil'man [25]. Over the last decades there has been a significant development of this theory, but in spite of its importance, this development has been quite slow due to the special features of impulsive differential equations in general, such as pulse phenomena, confluence, and loss of autonomy (see, for instance, [10]). An impulsive differential equation is described by three components: a continuous-time differential equation, which governs the state of the system between impulses; an impulse equation, which models an impulsive jump defined by a jump function at the instant an impulse occurs; and a jump criterion, which defines a set of jump events in which the impulse equation is active (see [27]).

Recently, many researchers have studied the stability of stochastic differential equations using Lyapunov functions and obtained interesting results, for example, Liao [18], Mao [24], Caraballo et al. [7], Yang [32] amongst others. However, there are also several difficulties in the applications of the corresponding theories to specific problems. It is therefore necessary to seek some new methods to deal with the stability in order to overcome those difficulties.

To this end, Burton and other authors have applied the fixed point theory to investigate the stability of deterministic systems during the last years, and have obtained some more applicable conclusions which can be found, for example, in the monograph [5] and the works $[4,3,6,9,13,12,26,33]$. In addition, there are

some papers where the fixed point theory is used to investigate the stability of stochastic (delayed) differential equations (see for instance $[8,15,17,16,19,28$, $29,20,21,23,22])$. More precisely, in $[20,21,23]$ the authors used the fixed point theory to study the exponential stability of mild solutions for stochastic partial differential equations with bounded delays and with infinite delays. In $[28,29]$ the fixed point theory is used to discuss the asymptotic stability in $p$ th moment of mild solutions to nonlinear impulsive stochastic partial differential equations with bounded delays and infinite delays, and in [22] Luo used the fixed point theory to study the exponential stability of stochastic Volterra-Levin equations. Motivated by the works mentioned above, in this paper we study the mean square asymptotic stability of a class of impulsive neutral stochastic differential systems with variable delays by using a contraction mapping principle, and the obtained stability criteria are easily checked. In our result, the delays can be unbounded and the coefficients in the equations can change their sign. 
This paper is organized as follows. In Section 2, we present some basic preliminaries and the form of the impulsive neutral stochastic functional differential equations which will be studied. In Section 3, the main result concerning the existence of solutions and its mean square asymptotic stability will be stated and proved. In Section 4, an illustrative example is analyzed to test our results.

\section{Model description and preliminaries}

We consider the following class of impulsive neutral stochastic differential systems with variable delays:

$$
\begin{gathered}
d\left[u_{i}(t)-\sum_{j=1}^{n} q_{i j}(t) u_{j}\left(t-\tau_{j}(t)\right)\right]=\left[\sum_{j=1}^{n} a_{i j}(t) u_{j}(t)+\sum_{j=1}^{n} b_{i j}(t) f_{j}\left(u_{j}(t)\right)\right. \\
\left.+\sum_{j=1}^{n} c_{i j}(t) g_{j}\left(u_{j}\left(t-\delta_{j}(t)\right)\right)\right] d t+\sum_{j=1}^{n} \sigma_{i j}\left(u_{j}(t)\right) d w_{j}(t), t \geq t_{0}, t \neq t_{k}, \\
\Delta u_{i}\left(t_{k}\right)=u_{i}\left(t_{k}+0\right)-u_{i}\left(t_{k}\right)=I_{i k}\left(u_{i}\left(t_{k}\right)\right), k=1,2, \ldots
\end{gathered}
$$

This can be written in a vector-matrix form as follows:

$$
\begin{gathered}
d[u(t)-Q(t) u(t-\tau(t))]=[A(t) u(t)+B(t) f(u(t))+C(t) g(u(t-\delta(t))] d t \\
+\sigma(u(t)) d w(t), t \geq t_{0}, t \neq t_{k} \\
\Delta u_{i}\left(t_{k}\right)=u_{i}\left(t_{k}+0\right)-u_{i}\left(t_{k}\right)=I_{i k}\left(u_{i}\left(t_{k}\right)\right), k=1,2, \ldots
\end{gathered}
$$

for $i=1,2,3, \ldots, n$, where $u(t)=\left[u_{1}(t), u_{2}(t), . ., u_{n}(t)\right]^{T} \in \mathbb{R}^{n}$, and $a_{i j}, b_{i j}$, $c_{i j}, q_{i j} \in C\left(\mathbb{R}^{+}, \mathbb{R}\right)$, are continuous functions, $A(t)=\left(a_{i j}(t)\right)_{n \times n}, B(t)=$ $\left(b_{i j}(t)\right)_{n \times n}, Q(t)=\left(q_{i j}(t)\right)_{n \times n}$, are real matrices and $\sigma(\cdot)=\left(\sigma_{i j}(\cdot)\right)_{n \times n}$ is the diffusion coefficient matrix, $f(u(t))=\left[f_{1}\left(u_{1}(t)\right), f_{2}\left(u_{2}(t)\right), \ldots, f_{n}\left(u_{n}(t)\right)\right]^{T} \in$ $\mathbb{R}^{n}, g(u(t))=\left[g_{1}\left(u_{1}(t)\right), g_{2}\left(u_{2}(t)\right), \ldots, g_{n}\left(u_{n}(t)\right)\right]^{T} \in \mathbb{R}^{n}$, and $\tau_{j}, \delta_{j}, j=1, \ldots, n$, which are the variable delays, are continuous functions satisfying appropriate conditions described below.

Let $w(t)=\left[w_{1}(t), w_{2}(t), \ldots, w_{n}(t)\right]^{T}$ be an $n$-dimensional Brownian motion on the complete filtered probability space $\left(\Omega, \mathcal{F},\left\{\mathcal{F}_{t}\right\}_{t>0}, \mathbb{P}\right)$ where $\left\{\mathcal{F}_{t}\right\}_{t>0}$ is the natural filtration of $w(t)$ (i.e. $\mathcal{F}_{t}$ is the completion of $\sigma\{w(s): 0 \leq s \leq t\}$ ). The expresion $\Delta u_{i}\left(t_{k}\right)=u_{i}\left(t_{k}^{+}\right)-u_{i}\left(t_{k}^{-}\right)=I_{i k}\left(u_{i}\left(t_{k}\right)\right)$ denotes the impulse at moment $t_{k}$, and $t_{1}<t_{2}<\ldots$, is a strictly increasing sequence such that $t_{k}$ goes to infinity, $u_{i}\left(t_{k}^{+}\right)$and $u_{i}\left(t_{k}^{-}\right)$stand for the right-hand and the left-hand limits of $u_{i}(t)$ at the impulsive moment $t_{k}$ respectively. $I_{i k}\left(u_{i}\left(t_{k}\right)\right)$ shows the impulsive perturbation at the moment $t_{k}$. Here $C\left(S_{1}, S_{2}\right)$ denotes the set of all continuous functions $\varphi: S_{1} \rightarrow S_{2}$ with the supremum norm $\|$.$\| .$

Denote by $u(t)=u(t ; s, \varphi)=\left(u_{1}\left(t ; s, \varphi_{1}\right), \ldots, u_{n}\left(t ; s, \varphi_{2}\right)\right)^{T} \in \mathbb{R}^{n}$ the solution to (1) with the initial condition

$$
u_{i}(s)=\varphi_{i}(s) \text { for } s \in\left[m\left(t_{0}\right), t_{0}\right], \text { for each } t_{0} \geq 0,
$$


where

$$
\begin{gathered}
m_{j}\left(t_{0}\right)=\min \left\{\inf \left\{t-\tau_{j}(t), t \geq t_{0}\right\}, \inf \left\{t-\delta_{j}(t), t \geq t_{0}\right\}\right\} \\
m\left(t_{0}\right)=\min \left\{m_{j}\left(t_{0}\right), 1 \leq j \leq n\right\}
\end{gathered}
$$

and $\varphi_{i}(\cdot) \in C\left(\left[m\left(t_{0}\right), t_{0}\right], \mathbb{R}\right)$, and $s \rightarrow \varphi(s)=\left(\varphi_{1}(s), \ldots, \varphi_{n}(s)\right)^{T} \in \mathbb{R}^{n}$ belongs to the space $C\left(\left[m\left(t_{0}\right), t_{0}\right], \mathbb{R}^{n}\right)$, with the norm defined by $\|\varphi\|=$ $\sum_{i=1}^{n} \sup _{m\left(t_{0}\right) \leq s \leq t_{0}}\left|\varphi_{i}(s)\right|$. Finally, $\mathbb{E}$ will denote expectation.

Before proceeding, we firstly introduce some assumptions:

(A1) The delay functions $\tau_{j}, \delta_{j} \in C\left(\mathbb{R}^{+}, \mathbb{R}^{+}\right)$satisfy

$$
t-\delta_{j}(t) \rightarrow \infty \text { and } t-\tau_{j}(t) \rightarrow \infty \text { as } t \rightarrow \infty \text { for } j=1,2, \ldots, n .
$$

(A2) there exist nonnegative constants $\alpha_{j}$ such that for all $x, y \in \mathbb{R}$,

$$
\left|f_{j}(x)-f_{j}(y)\right| \leq \alpha_{j}|x-y|, j=1,2, \ldots, n .
$$

(A3) there exist nonnegative constants $\beta_{j}$ such that for all $x, y \in \mathbb{R}$,

$$
\left|g_{j}(x)-g_{j}(y)\right| \leq \beta_{j}|x-y|, j=1,2, \ldots, n .
$$

(A4) there exist nonnegative constants $L_{i j}$ such that for all $x, y \in \mathbb{R}$,

$$
\left|\sigma_{i j}(x)-\sigma_{i j}(y)\right| \leq L_{i j}|x-y|, i, j=1,2, \ldots, n .
$$

(A5) there exist nonnegative constants $p_{i k}$ such that for all $x, y \in \mathbb{R}$,

$$
\left|I_{i k}(x)-I_{i k}(y)\right| \leq p_{i k}|x-y|, i=1,2, \ldots, n, k=1,2, \ldots
$$

Throughout this paper, we always assume that

$$
f_{j}(0)=g_{j}(0)=I_{i j}(0)=\sigma_{i k}(0)=0, \text { for } i, j=1,2, \ldots, n, k=1,2 \ldots,
$$

which imply that problem (1) admits a trivial equilibrium $u=0$.

Very recently, Guo et al. published in [11] related results on the solutions of stochastic neutral differential equations (1) in the case no impulses are taken into account (i.e. $I_{i k}=0$ for all $i=1, \ldots, n, k=1,2, \ldots$ ). More precisely, the following result was established.

Theorem A. Suppose that assumptions (A1)-(A4) hold and there exist positive scalars $a_{i}$ such that, for any $t \geq 0$

$$
\begin{aligned}
& \sum_{i=1}^{n}\left\{\left[\sum _ { j = 1 } ^ { n } \left(\left|q_{i j}(t)\right|+\int_{0}^{t} e^{-a_{i}(t-s)}\left|\overline{a_{i j}}(s)\right| d s\right.\right.\right. \\
& +\int_{0}^{t} e^{-a_{i}(t-s)}\left|q_{i j}(s)\right| a_{i} d s+\int_{0}^{t} e^{-a_{i}(t-s)}\left|b_{i j}(s)\right| \alpha_{j} d s \\
& \left.\left.\left.+\int_{0}^{t} e^{-a_{i}(t-s)}\left|c_{i j}(s)\right| \beta_{j} d s\right)\right]^{2}+\frac{2}{a_{i}} \sum_{j=1}^{n} L_{i j}^{2}\right\} \leq \gamma<\frac{1}{2},
\end{aligned}
$$


where $\overline{a_{i j}}(t)=a_{i j}(t)(i \neq j), \overline{a_{i i}}(t)=a_{i i}(t)+a_{i}$. Then, for any $\varphi \in C\left(\left[m\left(t_{0}\right), t_{0}\right], \mathbb{R}^{n}\right)$ there exists a unique global solution $u\left(t, t_{0}, \varphi\right)$ of $(1)$ in the case $I_{i k}=0$ for all $i=1, \ldots, n, k=1,2, \ldots$ Moreover, the zero solution is mean-square asymptotically stable.

One of our main objectives in this paper is to improve Theorem A and to extend it to investigate a wider class of stochastic neutral differential equations with impulsive effects presented in (1) by proving a sufficient condition for the asymptotic stability of the zero solution.

For each $t_{0} \geq 0$ and $\varphi \in C\left(\left[m\left(t_{0}\right), t_{0}\right], \mathbb{R}^{n}\right)$ fixed, we define $X_{\varphi, t_{0}}^{l}=X_{\varphi_{1, t_{0}}}^{l_{1}} \times$ $X_{\varphi_{2}, t_{0}}^{l_{2}} \ldots \times X_{\varphi_{n, t_{0}}}^{l_{n}}$, where $l, l_{i}>0$ are positive numbers such that $l^{2}=\sum_{i=1}^{n} l_{i}^{2}$, and $X_{\varphi_{i, t_{0}}}^{l_{i}}$ is the space consisting of stochastic processes $u_{i}(\cdot, \cdot):\left[m\left(t_{0}\right), \infty\right) \times \Omega \rightarrow \mathbb{R}$ satisfying, for almost every $\omega \in \Omega$,

1) $u_{i}(\cdot, \omega)$ is continuous on $t \neq t_{k}(k=1,2, \ldots)$,

2) $\lim _{t \rightarrow t_{k}^{+}} u_{i}(t, \omega)$ and $\lim _{t \rightarrow t_{k}^{-}} u_{i}(t, \omega)$ exist; moreover,

$\lim _{t \rightarrow t^{-}} u_{i}(t, \omega)=u_{i}\left(t_{k}\right)$ for $k=1,2 \ldots$,

3) $u_{i}(s, \omega)=\varphi_{i}(s, \omega)$ on $s \in\left[m\left(t_{0}\right), t_{0}\right]$,

4) $\left\|u_{i}\right\|_{X_{\varphi_{i, t_{0}}}^{l_{i}}} \leq l_{i}$ for $t \geq t_{0}$ and $\mathbb{E}\left|u_{i}(t)\right|^{2} \rightarrow 0$ as $t \rightarrow \infty$, for $i=1,2, \ldots, n$, with $\left\|u_{i}\right\|_{X_{\varphi_{i, t_{0}}}^{l_{i}}}:=\left\{\mathbb{E}\left(\sup _{t \geq m\left(t_{0}\right)}\left|u_{i}(t)\right|^{2}\right)\right\}^{\frac{1}{2}}$.

We omit $\omega$ when no confusion is possible. Notice that $X_{\varphi, t_{0}}^{l}$ coincides with the space of all $\mathcal{F}_{t}$ - adapted processes $u(t, \omega):\left[m\left(t_{0}\right), \infty\right) \times \Omega \rightarrow \mathbb{R}^{n}$ which are almost surely continuous on $t \neq t_{k}, \lim _{t \rightarrow t_{k}^{+}} u(t, \omega)$ and $\lim _{t \rightarrow t_{k}^{-}} u(t, \omega)$ exist, and $\lim _{t \rightarrow t_{k}^{-}} u(t, \omega)=u\left(t_{k}\right), k=1,2 \ldots$ such that $u(t,)=.\varphi(t)$ on $t \in\left[m\left(t_{0}\right), t_{0}\right]$, and $\|u\|_{X} \leq l$ for $t \geq t_{0}, \mathbb{E} \sum_{i=1}^{n}\left|u_{i}(t)\right|^{2} \rightarrow 0$ as $t \rightarrow \infty$, if we consider the norm $\|u\|_{X}:=\left\{\sum_{i=1}^{n} \mathbb{E}\left(\sup _{t \geq m\left(t_{0}\right)}\left|u_{i}(t)\right|^{2}\right)\right\}^{\frac{1}{2}}$. It is clear that $X_{\varphi, t_{0}}^{l}$ is a complete metric space with metric induced by the norm $\|\cdot\|_{X}$.

When no confusion is possible we will not write $X_{\varphi, t_{0}}^{l}, X_{\varphi_{i, t_{0}}}^{l_{i}}$ but $X_{\varphi}^{l}, X_{\varphi_{i}}^{l_{i}}$ respectively.

Let us now recall the definitions of stability that will be used in the next section.

Definition 2.1: The zero solution of the system (1) is said to be :

i) stable if for any $\varepsilon>0$ and $t_{0} \geq 0$, there exists $\delta=\delta\left(\varepsilon, t_{0}\right)>0$ such that $\varphi \in C\left(\left[m\left(t_{0}\right), t_{0}\right], \mathbb{R}^{n}\right)$ and $\|\varphi\|<\delta$ imply $\mathbb{E} \sum_{i=1}^{n}\left|u_{i}\left(t, t_{0}, \varphi_{i}\right)\right|^{2}<\varepsilon$ for $t \geq t_{0}$.

ii) asymptotically stable if the zero solution is stable and for any $\varepsilon>0$ and $t_{0} \geq 0$, there exists $\delta=\delta\left(\varepsilon, t_{0}\right)>0$ such that $\varphi \in C\left(\left[m\left(t_{0}\right), t_{0}\right], \mathbb{R}^{n}\right)$ and 
$\|\varphi\|<\delta$ imply $\mathbb{E} \sum_{i=1}^{n}\left|u_{i}\left(t, t_{0}, \varphi_{i}\right)\right|^{2} \rightarrow 0$ as $t \rightarrow \infty$.

Definition 2.2: For any $t_{0} \geq 0$ and $\varphi \in C\left(\left[m\left(t_{0}\right), t_{0}\right], \mathbb{R}^{n}\right)$, a function $u(\cdot):=u\left(\cdot, t_{0}, \varphi\right):\left[m\left(t_{0}\right), \infty\right) \rightarrow \mathbb{R}^{n}$ is said to be a solution of $(1)$ on $\left[t_{0}, \infty\right)$ satisfying the initial value condition $u(t)=\varphi(t)$ for $t \in\left[m\left(t_{0}\right), t_{0}\right]$, if the following conditions are fulfilled:

i) $u(t)$ is absolutely continuous on $\left[t_{0}, t_{1}\right)$ and each interval $\left(t_{k}, t_{k+1}\right)$.

ii) $u\left(t_{k}^{+}\right)$and $u\left(t_{k}^{-}\right)$exist and $u\left(t_{k}^{-}\right)=u\left(t_{k}\right)$ for any $t_{k} \in\left[t_{0}, \infty\right)$.

iii) $u(t)$ satisfies (1) almost everywhere in $\left[t_{0}, \infty\right)$, and may have a discontinuity of the first kind at $t_{k}$ for $k=1,2, \ldots$

\section{Main Results}

As we mentioned previously, one of our objectives in this section is to generalize the work carried out in [11] to the case in which impulses are taken into account in the problem, and allowing the coefficients to be more general. In other words, we will establish and prove a sufficient condition ensuring the existence of solutions and the global asymptotic stability of the zero solution to equation (1). It is well known that studying the stability of an equation using a fixed point technique involves the construction of a suitable mapping possessing a fixed point. This can be an arduous task. Thus, in order to construct our mapping, we begin by transforming (1) into a more tractable, but equivalent, equation, which we will then invert to obtain an equivalent integral equation from which we derive a contractive mapping. After that, we define a suitable complete metric space, which may depend on the initial condition, so that using a contraction mapping principle, we can ensure the existence of a unique fixed point for this mapping, and hence a solution for (1), which in addition is proved automatically to be mean square asymptotically stable.

Now, we can state our main result.

Theorem 3.1. Suppose that assumptions (A1)-(A5) hold and there exist continuous functions $a_{i}:\left[t_{0}, \infty\right) \rightarrow \mathbb{R}^{+}$such that:

1) there exists a constant $\mu$ satisfying $\inf \left\{t_{k}-t_{k-1}\right\} \geq \mu$, for $k=1,2, \ldots$;

2) there exist constants $p_{i}$ such that $p_{i k} \leq p_{i} \mu$ for $i=1,2,3, \ldots, n$ and $k=1,2, \ldots$;

3) there exist constants $n_{i}>0$ such that $a_{i}(t) \geq n_{i}, t \in \mathbb{R}^{+}$for $i=$ $1,2,3, \ldots, n$;

4) for $t \in \mathbb{R}^{+}, t \geq t_{0}$ and a positive constant $\gamma<\frac{1}{4}$, the following inequality 
holds:

$$
\begin{aligned}
& \sum_{i=1}^{n}\left\{\left[\sum _ { j = 1 } ^ { n } \left(\left|q_{i j}(t)\right|+\int_{t_{0}}^{t} e^{-\int_{s}^{t} a_{i}(\xi) d \xi}\left|\overline{a_{i j}}(s)\right| d s\right.\right.\right. \\
& +\int_{t_{0}}^{t} e^{-\int_{s}^{t} a_{i}(\xi) d \xi}\left|q_{i j}(s)\right|\left|a_{i}(s)\right| d s+\int_{t_{0}}^{t} e^{-\int_{s}^{t} a_{i}(\xi) d \xi}\left|b_{i j}(s)\right| \alpha_{j} d s \\
& \left.\left.+\int_{t_{0}}^{t} e^{-\int_{s}^{t} a_{i}(\xi) d \xi}\left|c_{i j}(s)\right| \beta_{j} d s\right)\right]^{2} \\
& \left.+4 \sum_{j=1}^{n} \int_{t_{0}}^{t} L_{i j}^{2} e^{-2 \int_{t}^{s} a_{i}(\xi) d \xi} d s+p_{i}^{2}\left(\frac{1}{n_{i}}+\mu\right)^{2}\right\} \leq \gamma<\frac{1}{4},
\end{aligned}
$$

where $\overline{a_{i j}}(t)=a_{i j}(t)(i \neq j), \overline{a_{i i}}(t)=a_{i i}(t)+a_{i}(t)$. Then for any $\varphi \in$ $C\left(\left[m\left(t_{0}\right), t_{0}\right], \mathbb{R}^{n}\right)$ there exists a unique global solution $u\left(t, t_{0}, \varphi\right)$. Moreover, the zero solution of (1) is mean-square asymptotically stable.

Proof. First, by condition (3), we have

$$
\int_{0}^{t} a_{i}(\xi) d \xi \rightarrow \infty \text { as } t \rightarrow \infty
$$

For each $t_{0} \geq 0$, we set

$$
M_{i}=\sup _{t \geq 0}\left\{e^{-\int_{0}^{t} a_{i}(\xi) d \xi}\right\} .
$$

Unlike the procedure carried out in [11], where the authors used the variation of constants formula to rewrite the original equation as an integral equation, we have to proceed in a very different way which is motivated and justified by the appearance of the impulsive terms in our problem. This is a key feature for our subsequent work in this paper, and this is why it deserves a careful and detailed description. We rewrite (1) as

$$
\begin{aligned}
& d\left[u_{i}(t)-\sum_{j=1}^{n} q_{i j}(t) u_{j}\left(t-\tau_{j}(t)\right)\right] \\
= & {\left[-a_{i}(t) u_{i}(t)+\sum_{j=1}^{n} \overline{a_{i j}}(t) u_{j}(t)\right.} \\
& \left.+\sum_{j=1}^{n} b_{i j}(t) f_{j}\left(u_{j}(t)\right)+\sum_{j=1}^{n} c_{i j}(t) g_{j}\left(u_{j}\left(t-\delta_{j}(t)\right)\right)\right] d t \\
& +\sum_{j=1}^{n} \sigma_{i j}\left(u_{j}(t)\right) d w_{j}(t), t \geq t_{0}, t \neq t_{k}, \\
& \Delta u_{i}\left(t_{k}\right)=u_{i}\left(t_{k}+0\right)-u_{i}\left(t_{k}\right)=I_{i k}\left(u_{i}\left(t_{k}\right)\right), k=1,2, \ldots,
\end{aligned}
$$


with the intial condition

$$
u_{i}(t)=\varphi_{i}(t) \text { for } t \in\left[m\left(t_{0}\right), t_{0}\right] .
$$

Multiplying both sides of (14) by $e^{\int_{t_{0}}^{t} a_{i}(\xi) d \xi}$, for $t \geq t_{0}$ and $t \neq t_{k}$,

$$
\begin{aligned}
& d\left[e^{\int_{t_{0}}^{t} a_{i}(\xi) d \xi} u_{i}(t)\right] \\
= & e^{\int_{t_{0}}^{t} a_{i}(\xi) d \xi} d u_{i}(t)+a_{i}(t) u_{i}(t) e^{\int_{t_{0}}^{t} a_{i}(\xi) d \xi} \\
= & e^{\int_{t_{0}}^{t} a_{i}(\xi) d \xi}\left\{d\left(\sum_{j=1}^{n} q_{i j}(t) u_{j}\left(t-\tau_{j}(t)\right)\right)\right. \\
& \left.+\sum_{j=1}^{n} \overline{a_{i j}}(t) u_{j}(t)+\sum_{j=1}^{n} b_{i j}(t) f_{j}\left(u_{j}(t)\right)+\sum_{j=1}^{n} c_{i j}(t) g_{j}\left(u_{j}\left(t-\delta_{j}(t)\right)\right)\right\} d t \\
& +e^{\int_{t_{0}}^{t} a_{i}(\xi) d \xi} \sum_{j=1}^{n} \sigma_{i j}\left(u_{j}(t)\right) d w_{j}(t), \\
& \Delta u_{i}\left(t_{k}\right)=u_{i}\left(t_{k}+0\right)-u_{i}\left(t_{k}\right)=I_{i k}\left(u_{i}\left(t_{k}\right)\right), k=1,2, \ldots,
\end{aligned}
$$

and, after integrating from $t_{k-1}+\varepsilon(\varepsilon>0)$ to $t \in\left(t_{k-1}, t_{k}\right)(k=1,2, \ldots)$, we have

$$
\begin{aligned}
& u_{i}(t) e^{\int_{t_{0}}^{t} a_{i}(\xi) d \xi} \\
& =u_{i}\left(t_{k-1}+\varepsilon\right) e^{\int_{t_{0}-1}^{t_{k}+\varepsilon} a_{i}(\xi) d \xi}+\int_{t_{k-1}+\varepsilon}^{t} e^{\int_{t_{0}}^{s} a_{i}(\xi) d \xi} d\left(\sum_{j=1}^{n} q_{i j}(s) u_{j}\left(s-\tau_{j}(s)\right)\right) \\
& +\int_{t_{k-1}+\varepsilon}^{t} e^{\int_{t_{0}}^{s} a_{i}(\xi) d \xi} \sum_{j=1}^{n} \overline{a_{i j}}(s) u_{j}(s) d s+\int_{t_{k-1}+\varepsilon}^{t} e^{\int_{t_{0}}^{s} a_{i}(\xi) d \xi} \sum_{j=1}^{n} b_{i j}(s) f_{j}\left(u_{j}(s)\right) d s \\
& +\int_{t_{k-1}+\varepsilon}^{t} e^{\int_{t_{0}}^{s} a_{i}(\xi) d \xi} \sum_{j=1}^{n} c_{i j}(s) g_{j}\left(u_{j}\left(s-\delta_{j}(s)\right)\right) d s \\
& +\int_{t_{k-1}+\varepsilon}^{t} e^{\int_{t_{0}}^{s} a_{i}(\xi) d \xi} \sum_{j=1}^{n} \sigma_{i j}\left(u_{j}(s)\right) d w_{j}(s) \text {. }
\end{aligned}
$$


Letting $\varepsilon \rightarrow 0$ in $(15)$, we have for $t \in\left(t_{k-1}, t_{k}\right)(k=1,2, \ldots)$

$$
\begin{aligned}
& u_{i}(t) e^{\int_{t_{0}}^{t} a_{i}(\xi) d \xi} \\
= & u_{i}\left(t_{k-1}+0\right) e^{\int_{t_{0}}^{t_{k-1}} a_{i}(\xi) d \xi}+\int_{t_{k-1}}^{t} e^{\int_{t_{0}}^{s} a_{i}(\xi) d \xi} d\left(\sum_{j=1}^{n} q_{i j}(s) u_{j}\left(s-\tau_{j}(s)\right)\right) \\
& +\int_{t_{k-1}}^{t} e^{\int_{t_{0}}^{s} a_{i}(\xi) d \xi} \sum_{j=1}^{n} \overline{a_{i j}}(s) u_{j}(s) d s+\int_{t_{k-1}}^{t} e^{\int_{t_{0}}^{s} a_{i}(\xi) d \xi} \sum_{j=1}^{n} b_{i j}(s) f_{j}\left(u_{j}(s)\right) d s \\
& +\int_{t_{k-1}}^{t} e^{\int_{t_{0}}^{s} a_{i}(\xi) d \xi} \sum_{j=1}^{n} c_{i j}(s) g_{j}\left(u_{j}\left(s-\delta_{j}(s)\right)\right) d s \\
& +\int_{t_{k-1}}^{t} e^{\int_{t_{0}}^{s} a_{i}(\xi) d \xi} \sum_{j=1}^{n} \sigma_{i j}\left(u_{j}(s)\right) d w_{j}(s) .
\end{aligned}
$$

Setting $t=t_{k}-\varepsilon(\varepsilon>0)$ in $(16)$,

$$
\begin{aligned}
& u_{i}\left(t_{k}-\varepsilon\right) e^{\int_{t_{0}}^{t_{k}-\varepsilon} a_{i}(\xi) d \xi}=u_{i}\left(t_{k-1}+0\right) e^{\int_{t_{0}}^{t_{k-1}} a_{i}(\xi) d \xi} \\
& +\int_{t_{k-1}}^{t_{k}-\varepsilon} e^{\int_{t_{0}}^{s} a_{i}(\xi) d \xi} d\left(\sum_{j=1}^{n} q_{i j}(s) u_{j}\left(s-\tau_{j}(s)\right)\right) \\
& +\int_{t_{k-1}}^{t_{k}-\varepsilon} e^{\int_{t_{0}}^{s} a_{i}(\xi) d \xi} \sum_{j=1}^{n} \overline{a_{i j}}(s) u_{j}(s) d s+\int_{t_{k-1}}^{t_{k}-\varepsilon} e^{\int_{t_{0}}^{s} a_{i}(\xi) d \xi} \sum_{j=1}^{n} b_{i j}(s) f_{j}\left(u_{j}(s)\right) d s \\
& +\int_{t_{k-1}}^{t_{k}-\varepsilon} e^{\int_{t_{0}}^{s} a_{i}(\xi) d \xi} \sum_{j=1}^{n} c_{i j}(s) g_{j}\left(u_{j}\left(s-\delta_{j}(s)\right)\right) d s \\
& +\int_{t_{k-1}}^{t_{k}-\varepsilon} e^{\int_{t_{0}}^{s} a_{i}(\xi) d \xi} \sum_{j=1}^{n} \sigma_{i j}\left(u_{j}(s)\right) d w_{j}(s)
\end{aligned}
$$

and, letting $\varepsilon \rightarrow 0$, we obtain

$$
\begin{aligned}
& u_{i}\left(t_{k}-0\right) e^{\int_{t_{0}}^{t_{k}} a_{i}(\xi) d \xi} \\
= & u_{i}\left(t_{k-1}+0\right) e^{\int_{t_{0}}^{t_{k-1}} a_{i}(\xi) d \xi}+\int_{t_{k-1}}^{t_{k}} e^{\int_{t_{0}}^{s} a_{i}(\xi) d \xi} d\left(\sum_{j=1}^{n} q_{i j}(s) u_{j}\left(s-\tau_{j}(s)\right)\right) \\
& +\int_{t_{k-1}}^{t_{k}} e^{\int_{t_{0}}^{s} a_{i}(\xi) d \xi} \sum_{j=1}^{n} \overline{a_{i j}}(s) u_{j}(s) d s+\int_{t_{k-1}}^{t_{k}} e^{\int_{t_{0}}^{s} a_{i}(\xi) d \xi} \sum_{j=1}^{n} b_{i j}(s) f_{j}\left(u_{j}(s)\right) d s \\
& +\int_{t_{k-1}}^{t_{k}} e^{\int_{t_{0}}^{s} a_{i}(\xi) d \xi} \sum_{j=1}^{n} c_{i j}(s) g_{j}\left(u_{j}\left(s-\delta_{j}(s)\right)\right) d s \\
& +\int_{t_{k-1}}^{t_{k}} e^{\int_{t_{0}}^{s} a_{i}(\xi) d \xi} \sum_{j=1}^{n} \sigma_{i j}\left(u_{j}(s)\right) d w_{j}(s) .
\end{aligned}
$$


Noting $u_{i}\left(t_{k}-0\right)=u_{i}\left(t_{k}\right)$, (18) can be rearranged as

$$
\begin{aligned}
& u_{i}\left(t_{k}\right) e^{\int_{t_{0}}^{t_{k}} a_{i}(\xi) d \xi} \\
= & u_{i}\left(t_{k-1}+0\right) e^{\int_{t_{0}}^{t_{k-1}} a_{i}(\xi) d \xi}+\int_{t_{k-1}}^{t_{k}} e^{\int_{t_{0}}^{s} a_{i}(\xi) d \xi} d\left(\sum_{j=1}^{n} q_{i j}(s) u_{j}\left(s-\tau_{j}(s)\right)\right) \\
& +\int_{t_{k-1}}^{t_{k}} e^{\int_{t_{0}}^{s} a_{i}(\xi) d \xi} \sum_{j=1}^{n} \overline{a_{i j}}(s) u_{j}(s) d s+\int_{t_{k-1}}^{t_{k}} e^{\int_{t_{0}}^{s} a_{i}(\xi) d \xi} \sum_{j=1}^{n} b_{i j}(s) f_{j}\left(u_{j}(s)\right) d s \\
& +\int_{t_{k-1}}^{t_{k}} e^{\int_{t_{0}}^{s} a_{i}(\xi) d \xi} \sum_{j=1}^{n} c_{i j}(s) g_{j}\left(u_{j}\left(s-\delta_{j}(s)\right)\right) d s \\
& +\int_{t_{k-1}}^{t_{k}} e^{\int_{t_{0}}^{s} a_{i}(\xi) d \xi} \sum_{j=1}^{n} \sigma_{i j}\left(u_{j}(s)\right) d w_{j}(s) .
\end{aligned}
$$

Combining (16) and (19) we deduce

$$
\begin{aligned}
& u_{i}(t) e^{\int_{t_{0}}^{t} a_{i}(\xi) d \xi} \\
= & u_{i}\left(t_{k-1}+0\right) e^{\int_{t_{0}}^{t_{k-1}} a_{i}(\xi) d \xi}+\int_{t_{k-1}}^{t} e^{\int_{t_{0}}^{s} a_{i}(\xi) d \xi} d\left(\sum_{j=1}^{n} q_{i j}(s) u_{j}\left(s-\tau_{j}(s)\right)\right) d s \\
& +\int_{t_{k-1}}^{t} e^{\int_{t_{0}}^{s} a_{i}(\xi) d \xi} \sum_{j=1}^{n} \overline{a_{i j}}(s) u_{j}(s) d s+\int_{t_{k-1}}^{t} e^{\int_{t_{0}}^{s} a_{i}(\xi) d \xi} \sum_{j=1}^{n} b_{i j}(s) f_{j}\left(u_{j}(s)\right) d s \\
& +\int_{t_{k-1}}^{t} e^{\int_{t_{0}}^{s} a_{i}(\xi) d \xi} \sum_{j=1}^{n} c_{i j}(s) g_{j}\left(u_{j}\left(s-\delta_{j}(s)\right)\right) d s \\
& +\int_{t_{k-1}}^{t} e^{\int_{t_{0}}^{s} a_{i}(\xi) d \xi} \sum_{j=1}^{n} \sigma_{i j}\left(u_{j}(s)\right) d w_{j}(s),
\end{aligned}
$$

for $t \in\left(t_{k-1}, t_{k}\right](k=1,2, \ldots)$, and hence

$$
\begin{aligned}
& u_{i}(t) e^{\int_{t_{0}}^{t} a_{i}(\xi) d \xi}=\left\{u_{i}\left(t_{k-1}\right)+I_{i(k-1)}\left(u_{i}\left(t_{k-1}\right)\right)\right\} e^{\int_{t_{0}}^{t_{k-1}} a_{i}(\xi) d \xi} \\
& +\int_{t_{k-1}}^{t} e^{\int_{t_{0}}^{s} a_{i}(\xi) d \xi} d\left(\sum_{j=1}^{n} q_{i j}(s) u_{j}\left(s-\tau_{j}(s)\right)\right) d s \\
& +\int_{t_{k-1}}^{t} e^{\int_{t_{0}}^{s} a_{i}(\xi) d \xi} \sum_{j=1}^{n} \overline{a_{i j}}(s) u_{j}(s) d s+\int_{t_{k-1}}^{t} e^{\int_{t_{0}}^{s} a_{i}(\xi) d \xi} \sum_{j=1}^{n} b_{i j}(s) f_{j}\left(u_{j}(s)\right) d s \\
& +\int_{t_{k-1}}^{t} e^{\int_{t_{0}}^{s} a_{i}(\xi) d \xi} \sum_{j=1}^{n} c_{i j}(s) g_{j}\left(u_{j}\left(s-\delta_{j}(s)\right)\right) d s \\
& +\int_{t_{k-1}}^{t} e^{\int_{t_{0}}^{s} a_{i}(\xi) d \xi} \sum_{j=1}^{n} \sigma_{i j}\left(u_{j}(s)\right) d w_{j}(s)
\end{aligned}
$$




$$
\begin{aligned}
= & u_{i}\left(t_{k-1}\right) e^{\int_{t_{0}}^{t_{k-1}} a_{i}(\xi) d \xi}+\int_{t_{k-1}}^{t} e^{\int_{t_{0}}^{s} a_{i}(\xi) d \xi} d\left(\sum_{j=1}^{n} q_{i j}(s) u_{j}\left(s-\tau_{j}(s)\right)\right) d s \\
& +\int_{t_{k-1}}^{t} e^{\int_{t_{0}}^{s} a_{i}(\xi) d \xi} \sum_{j=1}^{n} \overline{a_{i j}}(s) u_{j}(s) d s+\int_{t_{k-1}}^{t} e^{\int_{t_{0}}^{s} a_{i}(\xi) d \xi} \sum_{j=1}^{n} b_{i j}(s) f_{j}\left(u_{j}(s)\right) d s \\
& +\int_{t_{k-1}}^{t} e^{\int_{t_{0}}^{s} a_{i}(\xi) d \xi} \sum_{j=1}^{n} c_{i j}(s) g_{j}\left(u_{j}\left(s-\delta_{j}(s)\right)\right) d s+\int_{t_{k-1}}^{t} e^{\int_{t_{0}}^{s} a_{i}(\xi) d \xi} \sum_{j=1}^{n} \sigma_{i j}\left(u_{j}(s)\right) d w_{j}(s) \\
& +I_{i(k-1)}\left(u_{i}\left(t_{k-1}\right)\right) e^{\int_{t_{0}}^{t_{k-1}}} a_{i}(\xi) d \xi
\end{aligned}
$$

which results in

$$
\begin{aligned}
& u_{i}\left(t_{k-1}\right) e^{\int_{t_{0}}^{t_{k-1}} a_{i}(\xi) d \xi} \\
& =u_{i}\left(t_{k-2}\right) e^{\int_{t_{0}-2}^{t_{k-2}} a_{i}(\xi) d \xi}+\int_{t_{k-2}}^{t_{k-1}} e^{\int_{t_{0}}^{s} a_{i}(\xi) d \xi} d\left(\sum_{j=1}^{n} q_{i j}(s) u_{j}\left(s-\tau_{j}(s)\right)\right) d s \\
& +\int_{t_{k-2}}^{t_{k-1}} e^{\int_{t_{0}}^{s} a_{i}(\xi) d \xi} \sum_{j=1}^{n} \overline{a_{i j}}(s) u_{j}(s) d s+\int_{t_{k-2}}^{t_{k-1}} e^{\int_{t_{0}}^{s} a_{i}(\xi) d \xi} \sum_{j=1}^{n} b_{i j}(s) f_{j}\left(u_{j}(s)\right) d s \\
& +\int_{t_{k-2}}^{t_{k-1}} e^{\int_{t_{0}}^{s} a_{i}(\xi) d \xi} \sum_{j=1}^{n} c_{i j}(s) g_{j}\left(u_{j}\left(s-\delta_{j}(s)\right)\right) d s+\int_{t_{k-2}}^{t_{k-1}} e^{\int_{t_{0}}^{s} a_{i}(\xi) d \xi} \sum_{j=1}^{n} \sigma_{i j}\left(u_{j}(s)\right) d w_{j}(s) \\
& +I_{i(k-2)}\left(u_{i}\left(t_{k-2}\right)\right) e^{\int_{t_{0}}^{t_{k-2}} a_{i}(\xi) d \xi}, \\
& u_{i}\left(t_{2}\right) e^{\int_{t_{0}}^{t_{2}} a_{i}(\xi) d \xi} \\
& =u_{i}\left(t_{1}\right) e^{\int_{t_{0}}^{t_{1}} a_{i}(\xi) d \xi}+\int_{t_{1}}^{t_{2}} e^{\int_{t_{0}}^{s} a_{i}(\xi) d \xi} d\left(\sum_{j=1}^{n} q_{i j}(s) u_{j}\left(s-\tau_{j}(s)\right)\right) d s \\
& +\int_{t_{1}}^{t_{2}} e^{\int_{t_{0}}^{s} a_{i}(\xi) d \xi} \sum_{j=1}^{n} \overline{a_{i j}}(s) u_{j}(s) d s+\int_{t_{1}}^{t_{2}} e^{\int_{t_{0}}^{s} a_{i}(\xi) d \xi} \sum_{j=1}^{n} b_{i j}(s) f_{j}\left(u_{j}(s)\right) d s \\
& +\int_{t_{1}}^{t_{2}} e^{\int_{t_{0}}^{s} a_{i}(\xi) d \xi} \sum_{j=1}^{n} c_{i j}(s) g_{j}\left(u_{j}\left(s-\delta_{j}(s)\right)\right) d s+\int_{t_{1}}^{t_{2}} e^{\int_{t_{0}}^{s} a_{i}(\xi) d \xi} \sum_{j=1}^{n} \sigma_{i j}\left(u_{j}(s)\right) d w_{j}(s) \\
& +I_{i 1}\left(u_{i}\left(t_{1}\right)\right) e^{\int_{t_{0}}^{t_{1}} a_{i}(\xi) d \xi}
\end{aligned}
$$


and

$$
\begin{aligned}
& u_{i}\left(t_{1}\right) e^{f_{t_{0}}^{t_{1}} a_{i}(\xi) d \xi} \\
= & u_{i}\left(t_{0}\right)+\int_{t_{0}}^{t_{1}} e^{\int_{t_{0}}^{s} a_{i}(\xi) d \xi} d\left(\sum_{j=1}^{n} q_{i j}(s) u_{j}\left(s-\tau_{j}(s)\right)\right) d s \\
& +\int_{t_{0}}^{t_{1}} e^{\int_{t_{0}}^{s} a_{i}(\xi) d \xi} \sum_{j=1}^{n} \overline{a_{i j}}(s) u_{j}(s) d s+\int_{t_{0}}^{t_{1}} e^{\int_{t_{0}}^{s} a_{i}(\xi) d \xi} \sum_{j=1}^{n} b_{i j}(s) f_{j}\left(u_{j}(s)\right) d s \\
& +\int_{t_{0}}^{t_{1}} e^{\int_{t_{0}}^{s} a_{i}(\xi) d \xi} \sum_{j=1}^{n} c_{i j}(s) g_{j}\left(u_{j}\left(s-\delta_{j}(s)\right)\right) d s \\
& +\int_{t_{0}}^{t_{1}} e^{\int_{t_{0}}^{s} a_{i}(\xi) d \xi} \sum_{j=1}^{n} \sigma_{i j}\left(u_{j}(s)\right) d w_{j}(s)+I_{i 0}\left(u_{i}\left(t_{0}\right)\right) .
\end{aligned}
$$

Performing an integration by parts, we can conclude, for $t \geq t_{0}, i=1,2, \ldots, n$,

$$
\begin{aligned}
& u_{i}(t) \\
= & {\left[u_{i}\left(t_{0}\right)-\left(\sum_{j=1}^{n} q_{i j}\left(t_{0}\right) u_{j}\left(t_{0}-\tau_{j}\left(t_{0}\right)\right)\right)\right] e^{-\int_{t_{0}}^{t} a_{i}(\xi) d \xi} } \\
& +\left(\sum_{j=1}^{n} q_{i j}(t) u_{j}\left(t-\tau_{j}(t)\right)\right) \\
& -\int_{t_{0}}^{t} a_{i}(s) e^{-\int_{s}^{t} a_{i}(\xi) d \xi}\left(\sum_{j=1}^{n} q_{i j}(s) u_{j}\left(s-\tau_{j}(s)\right)\right) d s \\
& +\int_{t_{0}}^{t} e^{-\int_{s}^{t} a_{i}(\xi) d \xi} \sum_{j=1}^{n} \overline{a_{i j}}(s) u_{j}(s) d s+\int_{t_{0}}^{t} e^{-\int_{s}^{t} a_{i}(\xi) d \xi} \sum_{j=1}^{n} b_{i j}(s) f_{j}\left(u_{j}(s)\right) d s \\
& +\int_{t_{0}}^{t} e^{-\int_{s}^{t} a_{i}(\xi) d \xi} \sum_{j=1}^{n} c_{i j}(s) g_{j}\left(u_{j}\left(s-\delta_{j}(s)\right)\right) d s \\
& +\int_{t_{0}}^{t} e^{-\int_{s}^{t} a_{i}(\xi) d \xi} \sum_{j=1}^{n} \sigma_{i j}\left(u_{j}(s)\right) d w_{j}(s) \\
& +e^{-\int_{t_{0}}^{t} a_{i}(\xi) d \xi} \sum_{t_{0}<t_{k}<t}^{n}\left\{I_{i k}\left(u_{i}\left(t_{k}\right)\right) e^{\int_{t_{0}}^{t_{k}} a_{i}(\xi) d \xi}\right\}
\end{aligned}
$$

Now, for a fixed initial function $\varphi:\left[m\left(t_{0}\right), t_{0}\right] \rightarrow \mathbb{R}^{n}$ with $\|\varphi\|=\delta_{0}$, we choose $l>\delta_{0}$ (and corresponding $l_{i}$ as described previously), such that

$$
4 \delta_{0} \sum_{i=1}^{n}\left(1+\sum_{j=1}^{n}\left|q_{i j}\left(t_{0}\right)\right|\right)^{2} M_{i}^{2} \leq l^{2}(1-4 \gamma) .
$$


For such initial function $\varphi$ and constants $l, l_{i}$ we consider the space $X_{\varphi}^{l}$ and define the operator $\mathcal{P}: X_{\varphi}^{l} \rightarrow X_{\varphi}^{l}$ by $(\mathcal{P} u)(t):=\left[\left(\mathcal{P}_{1} u_{1}\right)(t),\left(\mathcal{P}_{2} u_{2}\right)(t), \ldots,\left(\mathcal{P}_{n} u_{n}\right)(t)\right]^{T}$ $\in X_{\varphi}^{l}$, where $\mathcal{P}_{i}: X_{\varphi_{i}}^{l_{i}} \rightarrow X_{\varphi_{i}}^{l_{i}}$ satisfies $\left(\mathcal{P}_{i} u_{i}\right)(t)=\varphi_{i}(t)$ for $t \in\left[m\left(t_{0}\right), t_{0}\right]$, and for $t \geq t_{0}, \mathcal{P}_{i}\left(u_{i}\right):\left[m\left(t_{0}\right),+\infty\right) \rightarrow \mathbb{R}(i=1,2, \ldots, n)$ is defined as follows:

$$
\begin{aligned}
& \left(\mathcal{P}_{i} u_{i}\right)(t) \\
= & {\left[\varphi_{i}\left(t_{0}\right)-\left(\sum_{j=1}^{n} q_{i j}\left(t_{0}\right) \varphi_{j}\left(t_{0}-\tau_{j}\left(t_{0}\right)\right)\right)\right] e^{-\int_{t_{0}}^{t} a_{i}(\xi) d \xi} } \\
& +\left(\sum_{j=1}^{n} q_{i j}(t) u_{j}\left(t-\tau_{j}(t)\right)\right) \\
& -\int_{t_{0}}^{t} a_{i}(s) e^{-\int_{s}^{t} a_{i}(\xi) d \xi}\left(\sum_{j=1}^{n} q_{i j}(s) u_{j}\left(s-\tau_{j}(s)\right)\right) d s \\
& +\int_{t_{0}}^{t} e^{-\int_{s}^{t} a_{i}(\xi) d \xi} \sum_{j=1}^{n} \overline{a_{i j}}(s) u_{j}(s) d s \\
& +\int_{t_{0}}^{t} e^{-\int_{s}^{t} a_{i}(\xi) d \xi} \sum_{j=1}^{n} b_{i j}(s) f_{j}\left(u_{j}(s)\right) d s \\
& +\int_{t_{0}}^{t} e^{-\int_{s}^{t} a_{i}(\xi) d \xi} \sum_{j=1}^{n} c_{i j}(s) g_{j}\left(u_{j}\left(s-\delta_{j}(s)\right)\right) d s \\
& +\int_{t_{0}}^{t} e^{-\int_{s}^{t} a_{i}(\xi) d \xi} \sum_{j=1}^{n} \sigma_{i j}\left(u_{j}(s)\right) d w_{j}(s) \\
& +e^{-\int_{t_{0}}^{t} a_{i}(\xi) d \xi}\left(\sum_{t_{0}<t_{k}<t} I_{i k}\left(u_{i}\left(t_{k}\right)\right) e^{-\int_{t_{k}}^{t_{0}} a_{i}(\xi) d \xi}\right)=\sum_{m=1}^{8} Q_{i m}(t)
\end{aligned}
$$

where,

$$
\begin{aligned}
& Q_{i 1}(t)=\left[\varphi_{i}\left(t_{0}\right)-\left(\sum_{j=1}^{n} q_{i j}\left(t_{0}\right) \varphi_{j}\left(t_{0}-\tau_{j}\left(t_{0}\right)\right)\right)\right] e^{-\int_{t_{0}}^{t} a_{i}(\xi) d \xi} \\
& Q_{i 2}(t)=\sum_{j=1}^{n} q_{i j}(t) u_{j}\left(t-\tau_{j}(t)\right) \\
& Q_{i 3}(t)=\int_{t_{0}}^{t} a_{i}(s) e^{-\int_{s}^{t} a_{i}(\xi) d \xi}\left(\sum_{j=1}^{n} q_{i j}(s) u_{j}\left(s-\tau_{j}(s)\right)\right) d s \\
& Q_{i 4}(t)=\int_{t_{0}}^{t} e^{-\int_{s}^{t} a_{i}(\xi) d \xi} \sum_{j=1}^{n} \overline{a_{i j}}(s) u_{j}(s) d s
\end{aligned}
$$




$$
\begin{aligned}
& Q_{i 5}(t)=\int_{t_{0}}^{t} e^{-\int_{s}^{t} a_{i}(\xi) d \xi} \sum_{j=1}^{n} b_{i j}(s) f_{j}\left(u_{j}(s)\right) d s, \\
& Q_{i 6}(t)=\int_{t_{0}}^{t} e^{-\int_{s}^{t} a_{i}(\xi) d \xi} \sum_{j=1}^{n} c_{i j}(s) g_{j}\left(u_{j}\left(s-\delta_{j}(s)\right)\right) d s, \\
& Q_{i 7}(t)=\int_{t_{0}}^{t} e^{-\int_{s}^{t} a_{i}(\xi) d \xi} \sum_{j=1}^{n} \sigma_{i j}\left(u_{j}(s)\right) d w_{j}(s), \\
& Q_{i 8}(t)=e^{-\int_{t_{0}}^{t} a_{i}(\xi) d \xi}\left(\sum_{t_{0}<t_{k}<t} I_{i k}\left(u_{i}\left(t_{k}\right)\right) e^{-\int_{t_{k}}^{t_{0}} a_{i}(\xi) d \xi}\right) .
\end{aligned}
$$

To conclude the proof of our theorem, we proceed in three steps as follows.

First step: We prove that $\mathcal{P}\left(X_{\varphi}^{l}\right) \subset X_{\varphi}^{l}$. Let us first show the mean square continuity of $\mathcal{P}$ on $\left[t_{0}, \infty\right)$. For $u_{i} \in X_{\varphi_{i}}^{l_{i}}$, it is necessary to show that $\mathcal{P}_{i}\left(u_{i}\right) \in X_{\varphi_{i}}^{l_{i}}$. It is clear that $\mathcal{P}_{i}$ is continuous on $\left[m\left(t_{0}\right), t_{0}\right]$. For fixed time $t \geq t_{0}$, for each $i \in\{1,2,3, \ldots, n\}, u_{i} \in X_{\varphi_{i}}^{l_{i}}$, and $|\varepsilon|$ be sufficiently small,

$$
\mathbb{E}\left|\left(\mathcal{P}_{i}\left(u_{i}\right)\right)(t+\varepsilon)-\left(\mathcal{P}_{i}\left(u_{i}\right)\right)(t)\right|^{2} \leq 8 \sum_{m=1}^{8} \mathbb{E}\left|Q_{i m}(t+\varepsilon)-Q_{i m}(t)\right|^{2} .
$$

Since $u_{i} \in X_{\varphi_{i}}^{l_{i}}$, we know that $u_{i}$ is continuous on $t \neq t_{k}(k=1,2, \ldots)$. Moreover, $\lim _{t \rightarrow t_{k}^{-}} u_{i}(t)$ and $\lim _{t \rightarrow t_{k}^{+}} u_{i}(t)$ exist, in addition, $\lim _{t \rightarrow t_{k}^{-}} u_{i}(t)=u_{i}\left(t_{k}^{-}\right)$.

Letting $t \neq t_{k}(k=1,2, \ldots)$ in $(24)$, it is easy to see that,

$$
\mathbb{E}\left|Q_{i m}(t+\varepsilon)-Q_{i m}(t)\right|^{2} \rightarrow 0,(m=1,2, \ldots, 8), \text { as } \varepsilon \rightarrow 0,
$$

for $t \geq t_{0}$ and $t \neq t_{k}(k=1,2, \ldots)$.

Letting $t=t_{k}(k=1,2, \ldots)$ in $(24)$, it can be easily obtained

$$
\mathbb{E}\left|Q_{i m}(t+\varepsilon)-Q_{i m}(t)\right|^{2} \rightarrow 0 \text { as } \varepsilon \rightarrow 0 \text { for } m=1,2, \ldots, 7 .
$$

Letting $\varepsilon<0$ such that $|\varepsilon|$ is small enough, we have

$$
\begin{aligned}
& \mathbb{E}\left|Q_{i 8}\left(t_{k}+\varepsilon\right)-Q_{i 8}\left(t_{k}\right)\right|^{2} \\
& =\mathbb{E} \mid e^{-\int_{t_{0}}^{t_{k}+\varepsilon} a_{i}(\xi) d \xi} \sum_{t_{0}<t_{m}<t_{k}+\varepsilon}\left\{I_{i m}\left(u_{i}\left(t_{m}\right)\right) e^{-\int_{t_{m}}^{t_{0}} a_{i}(\xi) d \xi}\right\} \\
& -\left.e^{-\int_{t_{0}}^{t_{k}} a_{i}(\xi) d \xi} \sum_{t_{0}<t_{m}<t_{k}}\left\{I_{i m}\left(u_{i}\left(t_{m}\right)\right) e^{-\int_{t_{m}}^{t_{0}} a_{i}(\xi) d \xi}\right\}\right|^{2} \\
& =\left(e^{-\int_{t_{0}}^{t_{k}+\varepsilon} a_{i}(\xi) d \xi}-e^{-\int_{t_{0}}^{t_{k}} a_{i}(\xi) d \xi}\right)^{2} \mathbb{E}\left|\sum_{t_{0}<t_{m}<t_{k}}\left\{I_{i m}\left(u_{i}\left(t_{m}\right)\right) e^{-\int_{t_{m}}^{t_{0}} a_{i}(\xi) d \xi}\right\}\right|^{2},
\end{aligned}
$$

which implies $\lim _{\varepsilon \rightarrow 0^{-}} \mathbb{E}\left|Q_{i 8}\left(t_{k}+\varepsilon\right)-Q_{i 8}\left(t_{k}\right)\right|^{2}=0$.

Letting $\varepsilon>0$ be small enough, we have

$$
\begin{aligned}
& \mathbb{E}\left|Q_{i 8}\left(t_{k}+\varepsilon\right)-Q_{i 8}\left(t_{k}\right)\right|^{2} \\
& =\mathbb{E} \mid e^{-\int_{t_{0}}^{t_{k}+\varepsilon} a_{i}(\xi) d \xi} \sum_{t_{0}<t_{m}<t_{k}+\varepsilon}\left\{I_{i m}\left(u_{i}\left(t_{m}\right)\right) e^{-\int_{t_{m}}^{t_{0}} a_{i}(\xi) d \xi}\right\}
\end{aligned}
$$




$$
\begin{aligned}
& -\left.e^{-\int_{t_{0}}^{t_{k}} a_{i}(\xi) d \xi} \sum_{t_{0}<t_{m}<t_{k}}\left\{I_{i m}\left(u_{i}\left(t_{m}\right)\right) e^{-\int_{t_{m}}^{t_{0}} a_{i}(\xi) d \xi}\right\}\right|^{2} \\
& =\mathbb{E} \mid e^{-\int_{t_{0}}^{t_{k}+\varepsilon} a_{i}(\xi) d \xi}\left(\sum_{t_{0}<t_{m}<t_{k}}\left\{I_{i m}\left(u_{i}\left(t_{m}\right)\right) e^{-\int_{t_{m}}^{t_{0}} a_{i}(\xi) d \xi}\right\}+I_{i k}\left(u_{i}\left(t_{k}\right)\right) e^{-\int_{t_{k}}^{t_{0}} a_{i}(\xi) d \xi}\right) \\
& -\left.e^{-\int_{t_{0}}^{t_{k}} a_{i}(\xi) d \xi} \sum_{t_{0}<t_{m}<t_{k}}\left\{I_{i m}\left(u_{i}\left(t_{m}\right)\right) e^{-\int_{t_{m}}^{t_{0}} a_{i}(\xi) d \xi}\right\}\right|^{2} \\
& =\mathbb{E} \mid\left\{e^{-\int_{t_{0}}^{t_{k}+\varepsilon} a_{i}(\xi) d \xi}-e^{-\int_{t_{0}}^{t_{k}} a_{i}(\xi) d \xi}\right\} \sum_{t_{0}<t_{m}<t_{k}}\left\{I_{i m}\left(u_{i}\left(t_{m}\right)\right) e^{-\int_{t_{m}}^{t_{0}} a_{i}(\xi) d \xi}\right\} \\
& +\left.e^{-\int_{t_{0}}^{t_{k}+\varepsilon} a_{i}(\xi) d \xi} I_{i k}\left(u_{i}\left(t_{k}\right)\right) e^{-\int_{t_{k}}^{t_{0}} a_{i}(\xi) d \xi}\right|^{2} \\
& =\mathbb{E}\left|e^{-\int_{t_{0}}^{t_{k}} a_{i}(\xi) d \xi} I_{i k}\left(u_{i}\left(t_{k}\right)\right) e^{-\int_{t_{k}}^{t_{0}} a_{i}(\xi) d \xi}\right|^{2},
\end{aligned}
$$

which implies that

$$
\lim _{\varepsilon \rightarrow 0^{+}} \mathbb{E}\left|Q_{i 8}\left(t_{k}+\varepsilon\right)-Q_{i 8}\left(t_{k}\right)\right|^{2}=\mathbb{E}\left|I_{i k}\left(u_{i}\left(t_{k}\right)\right)\right|^{2} .
$$

According to the above discussion, we see that $\mathcal{P}_{i}\left(u_{i}\right):\left[m\left(t_{0}\right),+\infty\right) \rightarrow \mathbb{R}$ $(i=1,2, \ldots, n)$ is mean square continuous on $t \neq t_{k}(k=1,2, \ldots)$, while for $t=t_{k}(k=1,2, \ldots), \lim _{t \rightarrow t_{k}^{-}}\left(\mathcal{P}_{i}\left(u_{i}\right)\right)(t)$ and $\lim _{t \rightarrow t_{k}^{+}}\left(\mathcal{P}_{i}\left(u_{i}\right)\right)(t)$ exist. Furthermore, we also obtain that

$$
\lim _{t \rightarrow t_{k}^{-}}\left(\mathcal{P}_{i}\left(u_{i}\right)\right)(t)=\left(\mathcal{P}_{i}\left(u_{i}\right)\right)\left(t_{k}\right) \neq \lim _{t \rightarrow t_{k}^{+}}\left(\mathcal{P}_{i}\left(u_{i}\right)\right)(t) .
$$

Then we need to prove $\mathbb{E}\left|Q_{i m}(t)\right|^{2} \rightarrow 0$ as $t \rightarrow \infty$, for $m=1,2, \ldots, 8$. In fact, for any $\varepsilon>0$, there exists non-impulsive $T_{i}>0$, such that for $t \geq T_{i}$ we have $\mathbb{E}\left|u_{i}(t)\right|^{2}<\varepsilon$, for $i=1,2, \ldots, n$. Then, by condition (12), it is straightforward to prove

$$
\mathbb{E}\left|Q_{i 1}(t)\right|^{2} \leq \mathbb{E}\left|\varphi_{i}\left(t_{0}\right)-\sum_{j=1}^{n} q_{i j}\left(t_{0}\right) \varphi_{j}\left(t_{0}-\tau_{j}\left(t_{0}\right)\right)\right|^{2} e^{-2 \int_{t_{0}}^{t} a_{i}(\xi) d \xi} \rightarrow 0, \text { as }
$$
$t \rightarrow \infty$, which yields $\mathbb{E}\left|Q_{i 1}(t)\right|^{2} \rightarrow 0$ as $t \rightarrow \infty$.

Also, due to the fact that $\mathbb{E}\left|u_{j}(t)\right|^{2} \rightarrow 0$ and $t-\tau_{j}(t) \rightarrow \infty$ for $j=1,2, \ldots, n$, as $t \rightarrow \infty$, then

$$
\begin{aligned}
& \mathbb{E}\left|Q_{i 2}(t)\right|^{2} \leq \mathbb{E}\left|\sum_{j=1}^{n} q_{i j}(t) u_{j}\left(t-\tau_{j}(t)\right)\right|^{2} \\
& \leq n\left(\sum_{j=1}^{n}\left|q_{i j}(t)\right|^{2} \mathbb{E}\left|u_{j}\left(t-\tau_{j}(t)\right)\right|^{2}\right) \rightarrow 0,
\end{aligned}
$$

which means that $\mathbb{E}\left(\left|Q_{i 2}(t)\right|^{2}\right) \rightarrow 0$ as $t \rightarrow \infty$.

$$
\mathbb{E}\left|Q_{i 3}(t)\right|^{2} \leq \mathbb{E}\left|\int_{t_{0}}^{t} a_{i}(s) e^{-\int_{s}^{t} a_{i}(\xi) d \xi}\left(\sum_{j=1}^{n} q_{i j}(s) u_{j}\left(s-\tau_{j}(s)\right)\right) d s\right|^{2}
$$




$$
\begin{aligned}
& \leq \mathbb{E} \mid \int_{t_{0}}^{T} a_{i}(s) e^{-\int_{s}^{t} a_{i}(\xi) d \xi}\left(\sum_{j=1}^{n} q_{i j}(s) u_{j}\left(s-\tau_{j}(s)\right)\right) d s \\
& +\left.\int_{T}^{t} a_{i}(s) e^{-\int_{s}^{t} a_{i}(\xi) d \xi}\left(\sum_{j=1}^{n} q_{i j}(s) u_{j}\left(s-\tau_{j}(s)\right)\right) d s\right|^{2} \\
& \leq 2 \mathbb{E}\left(\sup _{\sigma>m\left(t_{0}\right)}\left|u_{j}(\sigma)\right|^{2}\right) \mathbb{E}\left|\int_{t_{0}}^{T} a_{i}(s) e^{-\int_{s}^{t} a_{i}(\xi) d \xi}\left(\sum_{j=1}^{n} q_{i j}(s)\right) d s\right|^{2} \\
& +2 \varepsilon \mathbb{E}\left|\int_{T}^{t} a_{i}(s) e^{-\int_{s}^{t} a_{i}(\xi) d \xi}\left(\sum_{j=1}^{n} q_{i j}(s)\right) d s\right|^{2} .
\end{aligned}
$$

By using condition (12), there is $T_{1} \geq T$ such that when $t \geq T_{1}$ we have

$$
2 \mathbb{E}\left(\sup _{\sigma>m\left(t_{0}\right)}\left|u_{j}(\sigma)\right|^{2}\right) e^{-2 \int_{T_{1}}^{t} a_{i}(\xi) d \xi}\left|\int_{t_{0}}^{T} a_{i}(s) e^{-\int_{s}^{T_{1}} a_{i}(\xi) d \xi}\left(\sum_{j=1}^{n} q_{i j}(s)\right) d s\right|^{2} \leq(1-\gamma) \varepsilon .
$$

By condition (11), $\mathbb{E}\left|Q_{i 3}(t)\right|^{2} \leq \gamma \varepsilon+(1-\gamma) \varepsilon=\varepsilon$. Thus $\mathbb{E}\left|Q_{i 3}(t)\right|^{2} \rightarrow 0$ as $t \rightarrow \infty$.

Similarly, we have that $\mathbb{E}\left(\left|Q_{i m}(t)\right|^{2}\right) \rightarrow 0(m=4,5,6)$ as $t \rightarrow \infty$.

$$
\begin{aligned}
& \mathbb{E}\left|Q_{i 7}(t)\right|^{2} \leq \mathbb{E} \int_{t_{0}}^{T} e^{-2 \int_{s}^{t} a_{i}(\xi) d \xi} \sum_{j=1}^{n}\left|\sigma_{i j}^{2}\left(u_{j}(s)\right)\right| d s \\
& +\mathbb{E} \int_{T}^{t} e^{-2 \int_{s}^{t} a_{i}(\xi) d \xi} \sum_{j=1}^{n}\left|\sigma_{i j}^{2}\left(u_{j}(s)\right)\right| d s \\
& \leq \sum_{j=1}^{n} L_{i j}^{2} \mathbb{E}\left(\sup _{s>m\left(t_{0}\right)}\left|u_{j}(s)\right|\right)^{2} e^{-2 \int_{T}^{t} a_{i}(\xi) d \xi}\left(\int_{t_{0}}^{T} e^{-2 \int_{s}^{t} a_{i}(\xi) d \xi} d s\right) \\
& +\varepsilon \sum_{j=1}^{n} L_{i j}^{2}\left(\int_{T}^{t} e^{-2 \int_{s}^{t} a_{i}(\xi) d \xi} d s\right) \\
& \leq \sum_{j=1}^{n} L_{i j}^{2} \mathbb{E}\left(\sup _{s>m\left(t_{0}\right)}\left|u_{j}(s)\right|\right)^{2} e^{-2 \int_{T_{1}}^{t} a_{i}(\xi) d \xi}\left(\int_{t_{0}}^{T_{1}} e^{-2 \int_{s}^{T_{1}} a_{i}(\xi) d \xi} d s\right)+\varepsilon \gamma .
\end{aligned}
$$

By using condition (12), there is $T_{1} \geq T$ such that when $t \geq T_{1}$ we have

$$
\sum_{j=1}^{n} L_{i j}^{2} \mathbb{E}\left(\sup _{s>m\left(t_{0}\right)}\left|u_{j}(s)\right|\right)^{2} e^{-2 \int_{T_{1}}^{t} a_{i}(\xi) d \xi}\left(\int_{t_{0}}^{T_{1}} e^{-2 \int_{s}^{T_{1}} a_{i}(\xi) d \xi} d s\right) \leq(1-\gamma) \varepsilon .
$$

Thanks to condition $(11), \mathbb{E}\left|Q_{i 7}(t)\right|^{2} \leq \gamma \varepsilon+(1-\gamma) \varepsilon=\varepsilon$. Thus $\mathbb{E}\left|Q_{i 7}(t)\right|^{2} \rightarrow$ 0 as $t \rightarrow \infty$.

$$
\begin{aligned}
& \mathbb{E}\left|Q_{i 8}(t)\right|^{2} \leq e^{-2 \int_{t_{0}}^{t} a_{i}(\xi) d \xi} \mathbb{E}\left|\sum_{t_{0}<t_{k}<t}\left\{p_{i k}\left|u_{i}\left(t_{k}\right)\right| e^{-\int_{t_{k}}^{t_{0}} a_{i}(\xi) d \xi}\right\}\right|^{2} \\
& \leq 2 e^{-2 \int_{t_{0}}^{t} a_{i}(\xi) d \xi} \mathbb{E}\left|\sum_{t_{0}<t_{k}<T_{i}}\left\{p_{i k}\left|u_{i}\left(t_{k}\right)\right| e^{-\int_{t_{k}}^{t_{0}} a_{i}(\xi) d \xi}\right\}\right|^{2}
\end{aligned}
$$




$$
\begin{aligned}
& +2 \varepsilon e^{-2 \int_{t_{0}}^{t} a_{i}(\xi) d \xi}\left|\sum_{T_{i}<t_{k}<t} p_{i k} e^{-\int_{t_{k}}^{t_{0}} a_{i}(\xi) d \xi}\right|^{2} \\
& \leq 2 e^{-2 \int_{t_{0}}^{t} a_{i}(\xi) d \xi} \mathbb{E}\left|\sum_{t_{0}<t_{k}<T_{i}}\left\{p_{i k}\left|u_{i}\left(t_{k}\right)\right| e^{-\int_{t_{k}}^{t_{0}} a_{i}(\xi) d \xi}\right\}\right|^{2} \\
& +2 \varepsilon e^{-2 \int_{t_{0}}^{t} a_{i}(\xi) d \xi}\left|\sum_{T_{i}<t_{k}<t} p_{i}\left(t_{k}-t_{k+1}\right) e^{-\int_{t_{k}}^{t_{0}} a_{i}(\xi) d \xi}\right|^{2} \\
& \leq 2 e^{-2 \int_{t_{0}}^{t} a_{i}(\xi) d \xi} \mathbb{E}\left|\sum_{t_{0}<t_{k}<T_{i}}\left\{p_{i k}\left|u_{i}\left(t_{k}\right)\right| e^{-\int_{t_{k}}^{t_{0}} a_{i}(\xi) d \xi}\right\}\right|^{2} \\
& +2 \varepsilon e^{-2 n_{i} t}\left|\sum_{T_{i}<t_{k}<t} p_{i}\left(t_{k}-t_{k+1}\right) e^{n_{i} t_{k}}\right|^{2} \\
& \leq 2 e^{-2 \int_{t_{0}}^{t} a_{i}(\xi) d \xi} \mathbb{E}\left|\sum_{t_{0}<t_{k}<T_{i}}\left\{p_{i k}\left|u_{i}\left(t_{k}\right)\right| e^{-\int_{t_{k}}^{t_{0}} a_{i}(\xi) d \xi}\right\}\right|^{2} \\
& +2 \varepsilon p_{i}^{2} e^{-2 n_{i} t}\left|\sum_{T_{i}<t_{r}<t_{k}}\left(t_{r+1-} t_{r}\right) e^{n_{i} t_{r}}+\mu e^{n_{i} t_{k}}\right|^{2} \\
& \leq 2 e^{-2 \int_{t_{0}}^{t} a_{i}(\xi) d \xi} \mathbb{E}\left|\sum_{t_{0}<t_{k}<T_{i}}\left\{p_{i k}\left|u_{i}\left(t_{k}\right)\right| e^{-\int_{t_{k}}^{t_{0}} a_{i}(\xi) d \xi}\right\}\right|^{2} \\
& +4 \varepsilon p_{i}^{2} e^{-2 n_{i} t}\left(\left|\int_{T_{i}}^{t} e^{n_{i} s} d s\right|^{2}+\left|\mu e^{n_{i} t}\right|^{2}\right) \\
& \leq 2 e^{-2 \int_{t_{0}}^{t} a_{i}(\xi) d \xi} \mathbb{E}\left|\sum_{t_{0}<t_{k}<T_{i}}\left\{p_{i k}\left|u_{i}\left(t_{k}\right)\right| e^{-\int_{t_{k}}^{t_{0}} a_{i}(\xi) d \xi}\right\}\right|^{2}+4 \varepsilon p_{i}^{2}\left(\frac{1}{n_{i}^{2}}+\mu^{2}\right) \\
& \leq 2 e^{-2 \int_{t_{0}}^{t} a_{i}(\xi) d \xi} \mathbb{E} \mid \sum_{t_{0}<t_{k}<T_{i}}\left\{\left.p_{i k}\left|u_{i}\left(t_{k}\right)\right| e^{-\int_{t_{k}}^{t_{0}} a_{i}(\xi) d \xi}\right|^{2}+4 \varepsilon \gamma .\right.
\end{aligned}
$$

By condition (12), there is $T_{1} \geq T_{i}$ such that for $t \geq T_{1}$

$\mathbb{E}\left(\sup _{t_{0}<t_{k}<T_{i}}\left|u_{i}\left(t_{k}\right)\right|\right)^{2} e^{-2 \int_{t_{0}}^{T_{1}} a_{i}(\xi) d \xi} e^{-2 \int_{T_{1}}^{t} a_{i}(\xi) d \xi}\left|\sum_{t_{0}<t_{k}<T_{i}}\left\{p_{i k} e^{-\int_{t_{k}}^{t_{0}} a_{i}(\xi) d \xi}\right\}\right|^{2} \leq$ $(1-4 \gamma) \varepsilon$.

which means that $\mathbb{E}\left(\left|Q_{i 8}(t)\right|^{2}\right) \rightarrow 0$ as $t \rightarrow \infty$. This yields $\mathbb{E}\left|\left(\mathcal{P}_{i}\left(u_{i}\right)\right)(t)\right|^{2} \rightarrow 0$ as $t \rightarrow \infty$.

Let us now prove that for any $u \in X_{\varphi}^{l}$ we have $\|\mathcal{P} u\|_{X} \leq l$. Since $f, g, \sigma$, $I_{i k}, i=1, \ldots, n, k=1,2, \ldots$ satisfy Lipschitz conditions, Eq. (25), condition (11) and the $L^{p}$-Doob imply

$$
\mathbb{E}\left[\sum_{i=1}^{n} \sup _{t \geq m\left(t_{0}\right)}\left|\left(\mathcal{P}_{i} u_{i}\right)(t)\right|^{2}\right]
$$




$$
\begin{aligned}
& \leq 4 \sum_{i=1}^{n}\left[\left|\varphi_{i}\left(t_{0}\right)\right|+\left(\sum_{j=1}^{n}\left|q_{i j}\left(t_{0}\right)\right|\left|\varphi_{j}\left(t_{0}-\tau_{j}\left(t_{0}\right)\right)\right|\right)\right]^{2} e^{-2 \int_{t_{0}}^{t} a_{i}(\xi) d \xi} \\
& +4 \sum_{i=1}^{n}\left\{\mathbb { E s u p } _ { t \geq t _ { 0 } } \left[\left(\sum_{j=1}^{n}\left|q_{i j}(t)\right|\left|u_{j}\left(t-\tau_{j}(t)\right)\right|\right)\right.\right. \\
& +\int_{t_{0}}^{t}\left|a_{i}(s)\right| e^{-\int_{s}^{t} a_{i}(\xi) d \xi}\left(\sum_{j=1}^{n}\left|q_{i j}(s)\right|\left|u_{j}\left(s-\tau_{j}(s)\right)\right|\right) d s \\
& +\int_{t_{0}}^{t} e^{-\int_{s}^{t} a_{i}(\xi) d \xi} \sum_{j=1}^{n}\left|\overline{a_{i j}}(s)\right|\left|u_{j}(s)\right| d s+\int_{t_{0}}^{t} e^{-\int_{s}^{t} a_{i}(\xi) d \xi} \sum_{j=1}^{n}\left|b_{i j}(s)\right|\left|f_{j}\left(u_{j}(s)\right)\right| d s \\
& \left.+\int_{t_{0}}^{t} e^{-\int_{s}^{t} a_{i}(\xi) d \xi} \sum_{j=1}^{n}\left|c_{i j}(s)\right|\left|g_{j}\left(u_{j}\left(s-\delta_{j}(s)\right)\right)\right| d s\right]^{2} \\
& +4 \sum_{i=1}^{n} \mathbb{E} \sup _{t \geq t_{0}}\left[\int_{t_{0}}^{t} e^{-\int_{s}^{t} a_{i}(\xi) d \xi} \sum_{j=1}^{n}\left|\sigma_{i j}\left(u_{j}(s)\right)\right| d w_{j}(s)\right]^{2} \\
& +4 \sum_{i=1}^{n} \mathbb{E} \sup _{t \geq t_{0}}\left[e^{-\int_{s}^{t} a_{i}(\xi) d \xi} \sum_{t_{0}<t_{k}<t}\left\{\left|I_{i k}\left(u_{i}\left(t_{k}\right)\right)\right| e^{-\int_{t_{k}}^{t_{0}} a_{i}(\xi) d \xi}\right\}\right]^{2} \\
& \leq 4 \sum_{i=1}^{n}\left[\left|\varphi_{i}\left(t_{0}\right)\right|^{2}\left(1+\sum_{j=1}^{n}\left|q_{i j}\left(t_{0}\right)\right|\right)^{2} e^{-2 \int_{t_{0}}^{t} a_{i}(\xi) d \xi}\right] \\
& \left.+4\left[\left.\sum_{i=1}^{n}\left(\mathbb{E} \sup _{s \geq m\left(t_{0}\right)} \mid u_{j}(s)\right)\right|^{2}\right)\right]\left\{\sum _ { i = 1 } ^ { n } \operatorname { s u p } _ { t \geq t _ { 0 } } \left[\left(\sum_{j=1}^{n}\left|q_{i j}(t)\right|\right)\right.\right. \\
& +\int_{t_{0}}^{t}\left|a_{i}(s)\right| e^{-\int_{s}^{t} a_{i}(\xi) d \xi}\left(\sum_{j=1}^{n}\left|q_{i j}(s)\right|\right) d s+\int_{t_{0}}^{t} e^{-\int_{s}^{t} a_{i}(\xi) d \xi} \sum_{j=1}^{n}\left|\overline{a_{i j}}(s)\right| d s \\
& \left.+\int_{t_{0}}^{t} e^{-\int_{s}^{t} a_{i}(\xi) d \xi} \sum_{j=1}^{n}\left|b_{i j}(s)\right| \alpha_{j} d s+\int_{t_{0}}^{t} e^{-\int_{s}^{t} a_{i}(\xi) d \xi} \sum_{j=1}^{n}\left|c_{i j}(s)\right| \beta_{j} d s\right]^{2} \\
& \left.+L_{i j}^{2} \int_{t_{0}}^{t} e^{-2 \int_{s}^{t} a_{i}(\xi) d \xi} d s+p_{i}^{2}\left(\frac{1}{n_{i}}+\mu\right)^{2}\right\} \\
& \left.\leq 4 \sum_{i=1}^{n}\left[\left|\varphi_{i}\left(t_{0}\right)\right|^{2}\left(1+\sum_{j=1}^{n}\left|q_{i j}\left(t_{0}\right)\right|\right)^{2} e^{-2 \int_{t_{0}}^{t} a_{i}(\xi) d \xi}\right]+4 \gamma\left[\left.\sum_{i=1}^{n}\left(\mathbb{E} \sup _{s \geq m\left(t_{0}\right)} \mid u_{j}(s)\right)\right|^{2}\right)\right] \\
& \leq 4 \delta_{0} \sum_{i=1}^{n}\left(1+\sum_{j=1}^{n}\left|q_{i j}\left(t_{0}\right)\right|\right)^{2} M_{i}^{2}+4 \gamma l^{2} \text {. }
\end{aligned}
$$




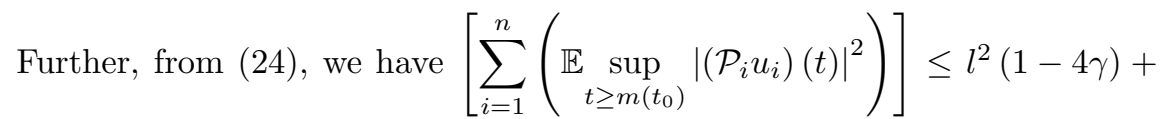
$4 \gamma l^{2}=l^{2}$. Hence $\|\mathcal{P} u\|_{X} \leq l$, and then $\mathcal{P}\left(X_{\varphi}^{l}\right) \subset X_{\varphi}^{l}$.

Second step: Now we will show that $\mathcal{P}$ has a unique fixed point $u$ in $X_{\varphi}^{l}$. For any $x=\left(x_{1}, x_{2}, \ldots, x_{n}\right)^{T} \in X_{\varphi}^{l}, y=\left(y_{1}, y_{2}, \ldots, y_{n}\right)^{T} \in X_{\varphi}^{l}$, we have

$$
\begin{aligned}
& \mathbb{E}\left(\sum_{i=1}^{n} \sup _{s \geq m\left(t_{0}\right)}\left|\left(\mathcal{P}_{i} x_{i}\right)(s)-\left(\mathcal{P}_{i} y_{i}\right)(s)\right|^{2}\right) \\
& \leq \mathbb{E}\left(\sum_{i=1}^{n} \sup _{s \geq t_{0}} \mid \sum_{j=1}^{n} q_{i j}(s)\left[x_{j}\left(s-\tau_{j}(s)\right)-y_{j}\left(s-\tau_{j}(s)\right)\right]\right. \\
& -\int_{t_{0}}^{s} a_{i}(u) e^{-\int_{s}^{t} a_{i}(\xi) d \xi} \sum_{j=1}^{n} q_{i j}(u)\left[x_{j}\left(u-\tau_{j}(u)\right)-y_{j}\left(u-\tau_{j}(u)\right)\right] d u \\
& +\int_{t_{0}}^{s} e^{-\int_{s}^{t} a_{i}(\xi) d \xi} \sum_{j=1}^{n} \overline{a_{i j}}(u)\left[x_{j}(u)-y_{j}(u)\right] d s \\
& +\int_{t_{0}}^{s} e^{-\int_{s}^{t} a_{i}(\xi) d \xi} \sum_{j=1}^{n} b_{i j}(u)\left[f_{j}\left(x_{j}(u)\right)-f_{j}\left(y_{j}(u)\right)\right] d u \\
& +\int_{t_{0}}^{s} e^{-\int_{s}^{t} a_{i}(\xi) d \xi} \sum_{j=1}^{n} c_{i j}(u)\left[g_{j}\left(x_{j}\left(u-\delta_{j}(u)\right)-g_{j}\left(y_{j}\left(u-\delta_{j}(u)\right)\right)\right)\right] d u \\
& +\int_{t_{0}}^{s} e^{-\int_{s}^{t} a_{i}(\xi) d \xi} \sum_{j=1}^{n}\left[\sigma_{i j}\left(x_{j}(u)\right)-\sigma_{i j}\left(y_{j}(u)\right)\right] d w_{j}(u) \\
& \left.+\left.e^{-\int_{t_{0}}^{t} a_{i}(\xi) d \xi} \sum_{t_{0}<t_{k}<s}\left\{\left[I_{i k}\left(x_{i}\left(t_{k}\right)-I_{i k}\left(y_{i}\left(t_{k}\right)\right)\right)\right] e^{-\int_{t_{k}}^{t_{0}} a_{i}(\xi) d \xi}\right\}\right|^{2}\right) .
\end{aligned}
$$

By using the Doob $L^{p}$-inequality (see [19]),

$$
\begin{aligned}
& \mathbb{E}\left[\sum_{i=1}^{n} \sup _{s \geq t_{0}}\left|\int_{t_{0}}^{s} e^{-\int_{s}^{t} a_{i}(\xi) d \xi} \sum_{j=1}^{n}\left[\sigma_{i j}\left(x_{j}(u)\right)-\sigma_{i j}\left(y_{j}(u)\right)\right] d w_{j}(u)\right|\right]^{2} \\
& \leq 4 \mathbb{E} \sum_{i=1}^{n} \sum_{j=1}^{n} \sup _{s \geq t_{0}}\left(\int_{t_{0}}^{s} e^{-2 \int_{s}^{t} a_{i}(\xi) d \xi}\left|\sigma_{i j}\left(x_{j}(u)\right)-\sigma_{i j}\left(y_{j}(u)\right)\right|^{2} d u\right) \\
& \left.\left.\leq\left. 4 \sum_{i=1}^{n} \sum_{j=1}^{n} L_{i j}^{2} \sup _{s \geq t_{0}}\left(\int_{t_{0}}^{s} e^{-2 \int_{s}^{t} a_{i}(\xi) d \xi} \mathbb{E} \sum_{j=1}^{n}\left(\sup _{u \geq m\left(t_{0}\right)} \mid x_{j}(u)\right)-y_{j}(u)\right)\right|^{2}\right) d u\right), \\
& \text { and } \\
& \mathbb{E}\left[e^{-\int_{t_{0}}^{t} a_{i}(\xi) d \xi} \sum_{t_{0}<t_{k}<s}\left\{\left|I_{i k}\left(x_{i}\left(t_{k}\right)\right)-I_{i k}\left(y_{i}\left(t_{k}\right)\right)\right| e^{-\int_{t_{k}}^{t_{0}} a_{i}(\xi) d \xi}\right\}\right]^{2} \\
& \leq \mathbb{E}\left[\sum_{i=1}^{n} \sup _{s \geq t_{0}}\left(e^{-n_{i} t} \sum_{t_{0}<t_{k}<s}\left\{\left|I_{i k}\left(x_{i}\left(t_{k}\right)\right)-I_{i k}\left(y_{i}\left(t_{k}\right)\right)\right| e^{n_{i} t_{k}}\right\}\right)\right]^{2}
\end{aligned}
$$




$$
\begin{aligned}
& \leq \mathbb{E} \sum_{j=1}^{n}\left(\sup _{s \geq m\left(t_{0}\right)}\left|x_{j}(s)-y_{j}(s)\right|^{2}\right) \times p_{i}^{2} e^{-2 n_{i} t}\left(\sum_{t_{0}<t_{k}<t} e^{n_{i} t_{k}} \mu\right)^{2} \\
& \leq\left[\mathbb{E} \sum_{j=1}^{n}\left(\sup _{s \geq m\left(t_{0}\right)}\left|x_{j}(s)-y_{j}(s)\right|^{2}\right)\right] \times p_{i}^{2} e^{-2 n_{i} t}\left(\sum_{t_{0}<t_{k}<t} e^{n_{i} t_{k}} \mu\right)^{2} \\
& \left.\left.\leq\left.\left[\mathbb{E} \sum_{j=1}^{n}\left(\sup _{s \geq m\left(t_{0}\right)} \mid x_{j}(s)\right)-y_{j}(s)\right)\right|^{2}\right)\right] \times \\
& \left.\left.\leq\left.\left[\mathbb{E} \sum_{j=1}^{n}\left(\sup _{s \geq m\left(t_{0}\right)} \mid x_{j}(s)\right)-y_{j}(s)\right)\right|^{2}\right)\right] \times p_{i}^{2} e^{-2 n_{i} t}\left(\int_{t_{0}}^{t} e^{n_{i} s} d s+e^{n_{i} t} \mu\right)^{2} \\
& \left.\left.\leq\left.\left[\mathbb{E} \sum_{j=1}^{n}\left(\sup _{s \geq m\left(t_{0}\right)} \mid x_{j}(s)\right)-y_{j}(s)\right)\right|^{2}\right)\right] \times p_{i} t_{i}\left(\frac{1}{n_{i}}+\mu\right)^{2} .
\end{aligned}
$$

Then,$$
\left\{\mathbb{E} \sum_{i=1}^{n} \sup _{s \geq m\left(t_{0}\right)}\left|\left(\mathcal{P}_{i} x_{i}\right)(s)-\left(\mathcal{P}_{i} y_{i}\right)(s)\right|^{2}\right\}^{\frac{1}{2}}
$$$$
\leq \sqrt{3}\left\{\left[\mathbb{E} \sum_{i=1}^{n}\left(\sup _{s \geq m\left(t_{0}\right)}\left|x_{i}(s)-y_{i}(s)\right|^{2}\right)\right]\right\}^{\frac{1}{2}}
$$$$
\times\left\{\sum _ { i = 1 } ^ { n } \operatorname { s u p } _ { s \geq t _ { 0 } } \left[\sum_{j=1}^{n}\left|q_{i j}(s)\right|+\int_{t_{0}}^{s} e^{-\int_{s}^{t} a_{i}(\xi) d \xi} \sum_{j=1}^{n}\left|a_{i}(u)\right|\left|q_{i j}(u)\right| d u\right.\right.
$$$$
+\int_{t_{0}}^{s} e^{-\int_{s}^{t} a_{i}(\xi) d \xi} \sum_{j=1}^{n}\left|\overline{a_{i j}}(u)\right| d s+\int_{t_{0}}^{s} e^{-\int_{s}^{t} a_{i}(\xi) d \xi} \sum_{j=1}^{n}\left|b_{i j}(u)\right| \alpha_{j} d u
$$$$
\left.+\int_{t_{0}}^{s} e^{-\int_{s}^{t} a_{i}(\xi) d \xi} \sum_{j=1}^{n}\left|c_{i j}(u)\right| \beta_{j} d u\right]^{2}
$$$$
\left.+4 \int_{t_{0}}^{s} e^{-2 \int_{s}^{t} a_{i}(\xi) d \xi} \sum_{j=1}^{n} L_{i j}^{2} d u+p_{i}^{2}\left(\frac{1}{n_{i}}+\mu\right)^{2}\right\}^{\frac{1}{2}} \text {. }
$$

By condition (11), $\mathcal{P}$ is a contraction mapping with constant $\sqrt{3 \gamma}$. Thanks to the contraction mapping principle (Smart [31], p. 2 ), we deduce that $\mathcal{P}$ : $X_{\varphi}^{l} \rightarrow X_{\varphi}^{l}$ possesses a unique fixed point in $X_{\varphi}^{l}$ which solves (1), is bounded and tends to zero as $t$ goes to infinity. Referring to [5], [9], [26], except for the fixed point method, we know of another way to prove that solutions of (1) are stable. Let $\varepsilon>0$ be given, by proceeding now in the opposite way as before, i.e., choosing a fixed $l=\varepsilon>0$, we obtain that there is $\delta>0$ ( small enough so that (24) holds with $\delta_{0}=\delta$ ) such that for $\|\varphi\|<\delta$ implies that the unique 
solution $u$ of (1) with $u=\varphi$ on $\left[m\left(t_{0}\right), t_{0}\right]$ satisfies $\mathbb{E} \sum_{i=1}^{n}\left|u_{i}\left(t, t_{0}, \varphi_{i}\right)\right|^{2}<\varepsilon$ for all $t \geq m\left(t_{0}\right)$. Moreover $\mathbb{E} \sum_{i=1}^{n}\left|u_{i}\left(t, t_{0}, \varphi_{i}\right)\right|^{2} \rightarrow 0$ as $t \rightarrow \infty$.

Third step: We will prove that the zero solution of (1) is mean-square asymptotically stable. Let $\varepsilon>0$ be given and choose $\delta>0(\delta<\varepsilon)$ satisfying

$$
4 \delta \sum_{i=1}^{n}\left(1+\sum_{j=1}^{n}\left|q_{i j}\left(t_{0}\right)\right|\right)^{2} M_{i}^{2}<(1-4 \gamma) \varepsilon,
$$

where $\gamma$ is the left hand side of (11). If $u(t)=u\left(t, t_{0}, \varphi\right)$ is a solution of (1) with the initial condition (3) satisfying $\|\varphi\|^{2}<\delta$, then $u(t)=(\mathcal{P} u)(t)$ as defined in (25). We claim that $\mathbb{E} \sum_{i=1}^{n}\left|u_{i}(t)\right|^{2}<\varepsilon$ for all $t \geq t_{0}$. Notice that $\mathbb{E} \sum_{i=1}^{n}\left|u_{i}(t)\right|^{2}<\varepsilon$ on $t \in\left[m\left(t_{0}\right), t_{0}\right]$, we suppose that there exists $t^{*}>t_{0}$ such that $\mathbb{E} \sum_{i=1}^{n}\left|u_{i}\left(t^{*}\right)\right|^{2}=\varepsilon$ and $\mathbb{E} \sum_{i=1}^{n}\left|u_{i}(t)\right|^{2}<\varepsilon$ for $m\left(t_{0}\right) \leq t \leq t^{*}$. Then, it follows from (25) and (27) that

$$
\begin{aligned}
& \mathbb{E} \sum_{i=1}^{n}\left|u_{i}\left(t^{*}\right)\right|^{2} \leq 4 \mathbb{E} \sum_{i=1}^{n}\left|\varphi_{i}\left(t_{0}\right)\right|^{2}\left[1+\sum_{j=1}^{n}\left|q_{i j}\left(t_{0}\right)\right|\right]^{2} e^{-2 \int_{t_{0}}^{t^{*}} a_{i}(\xi) d \xi} \\
& +4 \varepsilon \sum_{i=1}^{n}\left\{\left[\sum _ { j = 1 } ^ { n } \left(\left|q_{i j}\left(t^{*}\right)\right|+\int_{t_{0}}^{t^{*}} e^{-\int_{s}^{t^{*}} a_{i}(\xi) d \xi}\left|\overline{a_{i j}}(s)\right| d s\right.\right.\right. \\
& \left.\left.+\int_{t_{0}}^{t^{*}} e^{-\int_{s}^{t^{*}} a_{i}(\xi) d \xi}\left|q_{i j}(s)\right|\left|a_{i}(s)\right| d s\right)\right]^{2} \\
& \left.+L_{i j}^{2} \int_{t_{0}}^{t^{*}} e^{-2 \int_{s}^{t^{*}} a_{i}(\xi) d \xi} d s+p_{i}^{2}\left(\frac{1}{n_{i}}+\mu\right)^{2}\right\} \\
& \leq 4 \delta \sum_{i=1}^{n}\left(1+\sum_{j=1}^{n}\left|q_{i j}\left(t_{0}\right)\right|\right)^{2} e^{-2 \int_{t_{0}}^{t^{*}} a_{i}(\xi) d \xi}+4 \gamma \varepsilon \\
& \leq 4 \delta \sum_{i=1}^{n}\left(1+\sum_{j=1}^{n}\left|q_{i j}\left(t_{0}\right)\right|\right)^{2} M_{i}^{2}+4 \gamma \varepsilon<(1-4 \gamma) \varepsilon+4 \gamma \varepsilon=\varepsilon .
\end{aligned}
$$

which contradicts that $\mathbb{E} \sum_{i=1}^{n}\left|u_{i}\left(t^{*}\right)\right|^{2}=\varepsilon$. Thus $\mathbb{E} \sum_{i=1}^{n}\left|u_{i}(t)\right|^{2}<\varepsilon$ for all $t \geq t_{0}$, and the zero solution of (1) is stable. This shows that the zero solution of (1) is asymptotically stable. 
Remark 3.1: The results presented in this paper improve and extend the main result proved in Ref. [11] for non-impulsive cases.

Remark 3.2: It follows from the first part of the proof of Theorem 3.1 that the zero solution of (1) is stable under assumption (11). Moreover, Theorem 3.1 still holds true if (11) is satisfied for $t \geq t_{\rho}$ for some $t_{\rho} \in \mathbb{R}^{+}$.

\section{Example}

In this section, we analyze one example to illustrate the applicability of Theorem 3.1 .

Example: 4.1 Consider the following two-dimensional impulsive stochastic delay differential equation

$$
\begin{gathered}
d[x(t)-Q(t) x(t-\tau(t))]=[A(t) x(t)+B(t) f(x(t))] d t \\
+G(t) x(t) d w(t), t \geq 0, t \neq t_{k} \\
\Delta u_{i}\left(t_{k}\right)=u_{i}\left(t_{k}+0\right)-u_{i}\left(t_{k}\right)=0.1 u_{i}\left(t_{k}\right), k=1,2, \ldots
\end{gathered}
$$

where

$$
\begin{aligned}
& Q(t)=\left(\begin{array}{cc}
-\frac{\sin t}{10} & 0 \\
0.021 & \frac{\sin t}{50}
\end{array}\right), A(t)=\left(\begin{array}{cc}
-\frac{20}{1+e^{-t}} & 0 \\
0 & -\frac{40}{1+e^{-t}}
\end{array}\right) \\
& B(t)=\left(\begin{array}{cc}
-\frac{1}{\left(1+e^{-t}\right)^{2}} & 0 \\
-\frac{8}{5\left(1+e^{-t}\right)^{2}} & -\frac{1}{\left(1+e^{-t}\right)^{2}}
\end{array}\right), G(t)=\left(\begin{array}{cc}
\sqrt{\frac{1}{1+e^{-t}}} & 0 \\
0 & \sqrt{\frac{1}{2\left(1+e^{-t}\right)}}
\end{array}\right),
\end{aligned}
$$

$f_{j}(x(t))=\frac{|x+1|-|x-1|}{2}, j=1,2, p_{i k}\left(u_{i}\left(t_{k}\right)\right)=0.1 u_{i}\left(t_{k}\right)$ for $i=1,2$ and $k=$ $1,2, \ldots, t_{k}=t_{k+1}+0.2 k(k=1,2, \ldots)$. It is easy to see that $\mu=0.2$ and $\alpha_{j}=1$ as well as $p_{i k}=0.1$. If we choose $a_{1}(t)=\frac{20}{1+e^{-t}}, a_{2}(t)=\frac{40}{1+e^{-t}}$, and we select $p_{i}=0.5$.

By straightforward computations, we can check that condition (11) in Theorem 3.1 holds true, where $\tau \in C\left(\mathbb{R}^{+}, \mathbb{R}^{+}\right)$is an arbitrary continuous function which satisfies $t-\tau(t) \rightarrow \infty$ as $t \rightarrow \infty$. Then

$$
\begin{aligned}
& \sum_{i=1}^{n}\left\{\left[\sum _ { j = 1 } ^ { n } \left(\left|q_{i j}(t)\right|+\int_{0}^{t} e^{-\int_{s}^{t} a_{i}(\xi) d \xi}\left|\overline{a_{i j}}(s)\right| d s\right.\right.\right. \\
& \left.\left.+\int_{0}^{t} e^{-\int_{s}^{t} a_{i}(\xi) d \xi}\left|q_{i j}(s)\right|\left|a_{i}(s)\right| d s+\int_{0}^{t} e^{-\int_{s}^{t} a_{i}(\xi) d \xi}\left|b_{i j}(s)\right| \alpha_{j} d s\right)\right]^{2} \\
& \left.+4 \sum_{j=1}^{n} \int_{0}^{t} L_{i j}^{2} e^{-2 \int_{t}^{s} a_{i}(\xi) d \xi} d s+p_{i}^{2}\left(\frac{1}{n_{i}}+\mu\right)^{2}\right\} \leq 0.066+0.125+0.039<\frac{1}{4},
\end{aligned}
$$

and since $\exists n_{1}>0: a_{1}(t)>n_{1}$, and $\exists n_{2}>0: a_{2}(t)>n_{2}$, where $n_{1}=$ $10, n_{2}=20$, it is easy to see that all the conditions of Theorem 3.1 hold for $\gamma \simeq 0.23<\frac{1}{4}$. Thus, Theorem 3.1 implies that the zero solution of (28) is mean square asymptotically stable. 
Remark 4.1: From Example 4.1, we see that $a_{1}, a_{2}$ are not constant, whereas the functions $a_{1}, a_{2}$ in Ref. [11] are required to be constant. Therefore, the conditions in our paper improve those obtained in Ref. [11].

Acknowledgements. The research of the second author has been partially supported by grant MTM2015-63723-P (MINECO/FEDER, EU), and Junta de Andalucía (Spain) under the Proyecto de Excelencia P12-FQM-1492. Also, this work was funded by European Mathematical Society.

\section{References}

[1] Drumi Bainov and Pavel Simeonov. Oscillation theory of impulsive differential equations. International Publications, Orlando, FL, 1998.

[2] D. D. Bănov and P. S. Simeonov. Impulsive differential equations, volume 28 of Series on Advances in Mathematics for Applied Sciences. World Scientific Publishing Co., Inc., River Edge, NJ, 1995. Asymptotic properties of the solutions, Translated from the Bulgarian manuscript by $\mathrm{V}$. Covachev [V. Khr. Kovachev].

[3] T. A. Burton. Fixed points and stability of a nonconvolution equation. Proc. Amer. Math. Soc., 132(12):3679-3687, 2004.

[4] T. A. Burton. Fixed points, stability, and exact linearization. Nonlinear Anal., 61(5):857-870, 2005.

[5] T. A. Burton. Stability by fixed point theory for functional differential equations. Dover Publications, Inc., Mineola, NY, 2006.

[6] T. A. Burton and Bo Zhang. Fixed points and stability of an integral equation: nonuniqueness. Appl. Math. Lett., 17(7):839-846, 2004.

[7] Tomás Caraballo, Mohamed Ali Hammami, and Lassad Mchiri. Practical stability of stochastic delay evolution equations. Acta Appl. Math., 142:91$105,2016$.

[8] Guiling Chen, Onno van Gaans, and Sjoerd Verduyn Lunel. Fixed points and $p$ th moment exponential stability of stochastic delayed recurrent neural networks with impulses. Appl. Math. Lett., 27:36-42, 2014.

[9] Youssef M. Dib, Mariette R. Maroun, and Youssef N. Raffoul. Periodicity and stability in neutral nonlinear differential equations with functional delay. Electron. J. Differential Equations, pages No. 142, 11, 2005.

[10] L. H. Erbe and Xinzhi Liu. Existence results for boundary value problems of second order impulsive differential equations. J. Math. Anal. Appl., 149(1):56-69, 1990. 
[11] Yingxin Guo, Chao Xu, and Jun Wu. Stability analysis of neutral stochastic delay differential equations by a generalisation of Banach's contraction principle. Internat. J. Control, 90(8):1555-1560, 2017.

[12] Chuhua Jin and Jiaowan Luo. Fixed points and stability in neutral differential equations with variable delays. Proc. Amer. Math. Soc., 136(3):909918, 2008.

[13] Chuhua Jin and Jiaowan Luo. Stability in functional differential equations established using fixed point theory. Nonlinear Anal., 68(11):3307-3315, 2008 .

[14] V. Lakshmikantham, D. D. Baŭnov, and P. S. Simeonov. Theory of impulsive differential equations, volume 6 of Series in Modern Applied Mathematics. World Scientific Publishing Co., Inc., Teaneck, NJ, 1989.

[15] Xiaodi Li, Martin Bohner, and Chuan-Kui Wang. Impulsive differential equations: periodic solutions and applications. Automatica J. IFAC, 52:173-178, 2015.

[16] Xiaodi Li and Jinde Cao. An impulsive delay inequality involving unbounded time-varying delay and applications. IEEE Trans. Automat. Control, 62(7):3618-3625, 2017.

[17] Xiaodi Li and Jianhong Wu. Stability of nonlinear differential systems with state-dependent delayed impulses. Automatica J. IFAC, 64:63-69, 2016.

[18] X. X. Liao and X. Mao. Almost sure exponential stability of neutral stochastic differential difference equations. J. Math. Anal. Appl., 212(2):554-570, 1997.

[19] Jiaowan Luo. Fixed points and stability of neutral stochastic delay differential equations. J. Math. Anal. Appl., 334(1):431-440, 2007.

[20] Jiaowan Luo. Fixed points and exponential stability of mild solutions of stochastic partial differential equations with delays. J. Math. Anal. Appl., 342(2):753-760, 2008.

[21] Jiaowan Luo. Stability of stochastic partial differential equations with infinite delays. J. Comput. Appl. Math., 222(2):364-371, 2008.

[22] Jiaowan Luo. Fixed points and exponential stability for stochastic VolterraLevin equations. J. Comput. Appl. Math., 234(3):934-940, 2010.

[23] Jiaowan Luo and Takeshi Taniguchi. Fixed points and stability of stochastic neutral partial differential equations with infinite delays. Stoch. Anal. Appl., 27(6):1163-1173, 2009.

[24] Xuerong Mao, Yi Shen, and Chenggui Yuan. Almost surely asymptotic stability of neutral stochastic differential delay equations with Markovian switching. Stochastic Process. Appl., 118(8):1385-1406, 2008. 
[25] V. D. Mil man and A. D. Myškis. On the stability of motion in the presence of impulses. Sibirsk. Mat. Ž., 1:233-237, 1960.

[26] Y. N. Raffoul. Stability in neutral nonlinear differential equations with functional delays using fixed-point theory. Math. Comput. Modelling, 40(78):691-700, 2004.

[27] Alexander V. Roup, Dennis S. Bernstein, Sergey G. Nersesov, Wassim M. Haddad, and Vijaysekhar Chellaboina. Limit cycle analysis of the verge and foliot clock escapement using impulsive differential equations and Poincaré maps. Internat. J. Control, 76(17):1685-1698, 2003.

[28] R. Sakthivel and J. Luo. Asymptotic stability of impulsive stochastic partial differential equations with infinite delays. J. Math. Anal. Appl., 356(1):1-6, 2009 .

[29] R. Sakthivel and J. Luo. Asymptotic stability of nonlinear impulsive stochastic differential equations. Statist. Probab. Lett., 79(9):1219-1223, 2009 .

[30] A. M. Samoulenko and N. A. Perestyuk. Impulsive differential equations, volume 14 of World Scientific Series on Nonlinear Science. Series A: Monographs and Treatises. World Scientific Publishing Co., Inc., River Edge, NJ, 1995. With a preface by Yu. A. Mitropolskii and a supplement by S. I. Trofimchuk, Translated from the Russian by Y. Chapovsky.

[31] D. R. Smart. Fixed point theorems. Cambridge University Press, LondonNew York, 1974. Cambridge Tracts in Mathematics, No. 66.

[32] Jun Yang, Shouming Zhong, and Wenpin Luo. Mean square stability analysis of impulsive stochastic differential equations with delays. J. Comput. Appl. Math., 216(2):474-483, 2008.

[33] Bo Zhang. Fixed points and stability in differential equations with variable delays. Nonlinear Anal., 63(5-7):e233-e242, 2005. 